TRANSACTIONS OF THE

AMERICAN MATHEMATICAL SOCIETY

Volume 359, Number 10, October 2007, Pages 4729-4756

S 0002-9947(07)04344-9

Article electronically published on May 1, 2007

\title{
ON A SINGULAR PERTURBATION PROBLEM INVOLVING A "CIRCULAR-WELL" POTENTIAL
}

\author{
NELLY ANDRÉ AND ITAI SHAFRIR
}

\begin{abstract}
We study the asymptotic behavior, as a small parameter $\varepsilon$ goes to 0 , of the minimizers for a variational problem which involves a "circularwell" potential, i.e., a potential vanishing on a closed smooth curve in $\mathbb{R}^{2}$. We thus generalize previous results obtained for the special case of the GinzburgLandau potential.
\end{abstract}

\section{INTRODUCTION}

Let $\Gamma$ be a simple closed $C^{4}$-curve in $\mathbb{R}^{2}$ with length $l(\Gamma)$ which bounds a bounded domain $\Omega$. We define a "circular-well potential" as a function $W: \mathbb{R}^{2} \rightarrow$ $[0, \infty)$ satisfying

$$
W>0 \text { on } \mathbb{R}^{2} \backslash \Gamma \text { and } W=0 \text { on } \Gamma .
$$

We shall assume that $W$ too is of class $C^{4}$ and make two additional assumptions. Since $W$ attains its minimal value zero on $\Gamma$ we have clearly $W_{n}=0$ on $\Gamma$, where $W_{n}$ denotes the derivative in the direction of the exterior normal to $\partial \Omega=\Gamma$. We assume then that we are in the generic case, i.e., that

$$
W_{n n}>0 \text { on } \Gamma \text {. }
$$

Finally, we add a technical assumption on the behavior of $W$ at infinity: there exists $R_{0}>0$ such that

$$
\frac{\partial W}{\partial r} \geq 0 \text { for }|z|=r>R_{0} .
$$

Let $G$ be a simply connected, bounded domain in $\mathbb{R}^{2}$ with boundary of class $C^{2}$. For a given smooth boundary condition $g: \partial G \rightarrow \mathbb{R}^{2}$ (more assumptions will be imposed on $g$ later on) set

$$
H_{g}^{1}\left(G, \mathbb{R}^{2}\right)=\left\{u \in H^{1}\left(G, \mathbb{R}^{2}\right): u=g \text { on } \partial G\right\} .
$$

For every $\varepsilon>0$ let

$$
E_{\varepsilon}(u)=\int_{G}|\nabla u|^{2}+\frac{W(u(x))}{\varepsilon^{2}},
$$

and consider the minimization problem:

$$
\min \left\{E_{\varepsilon}(u): u \in H_{g}^{1}\left(G, \mathbb{R}^{2}\right)\right\} .
$$

Received by the editors April 5, 2005.

2000 Mathematics Subject Classification. Primary 35J20; Secondary 35B25, 35J60, 58E50.

Key words and phrases. Singular perturbation, circular-well potential, Ginzburg-Landau energy.

(C)2007 American Mathematical Society Reverts to public domain 28 years from publication 
We are interested in the asymptotic behavior of the minimizers $\left\{u_{\varepsilon}\right\}_{\varepsilon>0}$ and their energies $\left\{E_{\varepsilon}\left(u_{\varepsilon}\right)\right\}_{\varepsilon>0}$, as $\varepsilon \rightarrow 0$. In the case where $g$ is $\Gamma$-valued, this study can be done by using the methods developed by Bethuel, Brezis and Hélein in [4 and [5, and by Struwe in [12, for the case of the Ginzburg-Landau (GL) potential $W(z)=\left(1-|z|^{2}\right)^{2}$ (i.e., for $S^{1}$-valued $g$ ). Therefore, we shall focus on the case where $g$ is not necessarily $\Gamma$-valued.

Special cases of this problem were already studied by us in previous works. In 1 we considered the case of the GL-energy for $g$ which takes its values in $\mathbb{R}^{2} \backslash\{0\}$. In [3] we studied the more subtle case of the GL-energy with boundary condition which is allowed to have isolated zeros. In [2] we obtained the first result dealing with more general potentials, namely $W$ which is a function of the Euclidean distance to $\Gamma$ (with the assumption that the image of $g$ is "close enough" to $\Gamma$ ). The main object of the present article is to extend the results of [2] to the case of a general "circular-well" potential satisfying (1.1)-(1.3).

As we saw in [1, 3], the incompatibility of the boundary condition with the potential is responsible to the appearance of a boundary layer near $\partial G$, developed by the minimizer $u_{\varepsilon}$. This results in a contribution of the order $O\left(\frac{1}{\varepsilon}\right)$ to the energy $E_{\varepsilon}\left(u_{\varepsilon}\right)$. On the other hand, in the interior of the domain $G$ we find the "topological singularities" which contribute a term of the order $O(|\log \varepsilon|)$ to the energy. The main difficulty in this type of problem is to separate efficiently between these two contributions. In the case of the GL-energy (for simplicity we consider only nonvanishing $g$ ), this separation was quite easy to achieve, thanks to the following energy decomposition formula which is due to Mironescu and Lassoued [9]. Writing each admissible $u \in H^{1}\left(G, \mathbb{R}^{2}\right)$ as $u=\rho_{\varepsilon} v$, where $\rho_{\varepsilon}$ is the minimizer of the scalar GL-problem

$$
\min \left\{\int_{G}|\nabla \rho|^{2}+\frac{1}{\varepsilon^{2}}\left(1-\rho^{2}\right)^{2}: \rho \in H_{|g|}^{1}(G)\right\}
$$

we have

$\int_{G}|\nabla u|^{2}+\frac{1}{\varepsilon^{2}}\left(1-|u|^{2}\right)^{2}=\int_{G}\left|\nabla \rho_{\varepsilon}\right|^{2}+\frac{1}{\varepsilon^{2}}\left(1-\rho_{\varepsilon}^{2}\right)^{2}+\int_{G} \rho_{\varepsilon}^{2}|\nabla v|^{2}+\frac{1}{\varepsilon^{2}} \rho_{\varepsilon}^{4}\left(1-|v|^{2}\right)^{2}$.

The formula (1.6) was used in [1] to prove that

$$
u_{\varepsilon_{n}} \rightarrow u_{0}=e^{i \phi} \prod_{j=1}^{D} \frac{z-a_{j}}{\left|z-a_{j}\right|} \text { in } C_{\mathrm{loc}}^{k}\left(G \backslash\left\{a_{1}, \ldots, a_{D}\right\}\right),
$$

where $D$ is the absolute value of the degree of $g /|g|$. For a general potential $W$ we cannot expect an analogous formula to (1.6). Still, in the case treated in [3], where $W$ is a function of the distance to $\Gamma$, i.e., $W(u)=F(\operatorname{dist}(u, \Gamma))$, we do have a kind of an analogue to $\rho_{\varepsilon}$, given by the minimizer $d_{\varepsilon}$ to the problem

$$
\min \left\{\int_{G}|\nabla h|^{2}+\frac{F(h)}{\varepsilon^{2}}: h \in H^{1}(G), h(x)=\operatorname{dist}(g(x), \Gamma) \text { on } \partial G\right\} .
$$

It turns out that the energy of $d_{\varepsilon}$, i.e., the minimal value for the minimization problem (1.8), indeed gives the leading term in $E_{\varepsilon}\left(u_{\varepsilon}\right)$, of the order $O\left(\frac{1}{\varepsilon}\right)$. It is also useful in establishing a convergence result analogous to (1.7), but the analysis is much more involved than in the GL case (see [3] for details).

In the case of a potential $W$ satisfying only (1.1)-(1.3) we cannot associate to our problem any scalar minimization problem, like in (1.5) or (1.8), so new techniques 
are needed in order to separate between the two different types of contributions to the energy. A basic tool in the identification of the contribution from the boundary layer is a certain distance function to $\Gamma$, with respect to a degenerate Riemannian metric, associated with $W$. It is defined on $\mathbb{R}^{2}$ by

$$
\Psi(\zeta)=\inf _{\substack{\gamma \in \operatorname{Lip}\left([0,1], \mathbb{R}^{2}\right), \gamma(0) \in \Gamma, \gamma(1)=\zeta}} \int_{0}^{1}(W(\gamma(t)))^{1 / 2}\left|\gamma^{\prime}(t)\right| d t .
$$

Since the integral in (1.9) is invariant w.r.t. rescaling, we may replace the interval $[0,1]$ by any other closed interval. It is not difficult to see that $\Psi \in \operatorname{Lip}\left(\mathbb{R}^{2}\right)$ and that it is a solution of the eikonal-type equation

$$
|\nabla \Psi(\zeta)|^{2}=W(\zeta) \quad \text { a.e. on } \mathbb{R}^{2},
$$

with $\Psi=0$ on $\Gamma$. Functions of this type appeared in works on related problems by many authors, cf. Sternberg [11] and Fonseca-Tartar [7].

For each $\lambda>0$, the set $\Omega_{\lambda}=\left\{x \in \mathbb{R}^{2}: \Psi(x)<\lambda\right\}$ is a neighborhood of $\Gamma$. In Proposition 5.1 in the Appendix it is proved that $\Psi$ is of class $C^{2}$ in some neighborhood of $\Gamma$ (certainly it cannot have this regularity everywhere). Then, there exists a $\lambda_{0}>0$ satisfying

$$
\Psi \in C^{2}\left(\bar{\Omega}_{\lambda_{0}}\right) .
$$

When (1.11) holds, $\Omega_{\lambda_{0}}$ can be covered by a system of nonintersecting gradient lines of $\Psi$. In particular, for each $x \in \Omega_{\lambda_{0}}$ there exists a unique gradient line which passes through it, and we shall denote by $\tilde{s}(x)$ its intersection point with $\Gamma$. The map $\tilde{s}$ can be viewed as a projection from $\Omega_{\lambda_{0}}$ onto $\Gamma$, which is different, in general, from the Euclidean nearest point projection. We shall always assume that the smooth boundary condition $g: \partial G \rightarrow \mathbb{R}^{2}$ satisfies

$$
\text { Image }(g) \subset \Omega_{\lambda_{0}} \text {. }
$$

Therefore, by (1.11) (1.12) the map $\tilde{s}(g): \partial G \rightarrow \Gamma$ is continuous (actually it is even of class $C^{1}$ ), and we can use it to define the degree $D$ of $g$ by

$$
D=\operatorname{deg}(\tilde{s}(g), \partial G) .
$$

We shall assume in the sequel, without loss of generality, that $D \geq 0$. We denote

$$
\lambda_{1}:=\max \{\Psi(g(x)): x \in \partial G\},
$$

so that $0 \leq \lambda_{1}<\lambda_{0}$.

Our first result gives the asymptotic behavior of the energy of $u_{\varepsilon}$ as $\varepsilon$ goes to zero.

Theorem 1. Let $g: \partial G \rightarrow \mathbb{R}^{2}$ be a smooth map satisfying (1.12) of degree $D \geq 0$. Then,

$$
E_{\varepsilon}\left(u_{\varepsilon}\right)=\frac{2}{\varepsilon} \int_{\partial G} \Psi(g(\sigma)) d \sigma+D \frac{l^{2}(\Gamma)}{2 \pi} \log \frac{1}{\varepsilon}+O(1), \quad \forall \varepsilon>0 .
$$

The proof of the upper-bound in (1.15) is quite straightforward. On the other hand, the proof of the lower-bound is much more delicate. A key estimate is a 
lower-bound for the energy of $u_{\varepsilon}$ on $G_{c_{0} \varepsilon^{\alpha}}=\left\{x \in G\right.$ : $\left.\operatorname{dist}(x, \partial G) \leq c_{0} \varepsilon^{\alpha}\right\}$ for some $\alpha \in(1 / 2,1)$ and $c_{0}>0$ (see (3.30) below):

$$
\begin{aligned}
E_{\varepsilon}\left(u_{\varepsilon}, G_{c_{0} \varepsilon^{\alpha}}\right) & =\int_{G_{c_{0} \varepsilon^{\alpha}}}\left|\nabla u_{\varepsilon}\right|^{2}+\frac{W\left(u_{\varepsilon}\right)}{\varepsilon^{2}} \\
& \geq \frac{2}{\varepsilon} \int_{\partial G} \Psi(g(\sigma)) d \sigma+K \int_{\partial G} \frac{\left|u_{\varepsilon}\left(\sigma, c_{0} \varepsilon^{\alpha}\right)-\tilde{s}(g(\sigma))\right|^{2}}{\varepsilon^{\alpha}} d \sigma-C .
\end{aligned}
$$

Here we used the $(\sigma, \delta)$-coordinates where $\sigma(x)$ is the nearest point projection of $x$ on $\partial G$ and $\delta(x)=\operatorname{dist}(x, \partial G)$ (see Section 2 below). The second step consists of showing that

$$
E_{\varepsilon}\left(u_{\varepsilon}, G \backslash G_{c_{0} \varepsilon^{\alpha}}\right)+K \int_{\partial G} \frac{\left|u_{\varepsilon}\left(\sigma, c_{0} \varepsilon^{\alpha}\right)-\tilde{s}(g(\sigma))\right|^{2}}{\varepsilon^{\alpha}} d \sigma \geq D \frac{l^{2}(\Gamma)}{2 \pi} \log \frac{1}{\varepsilon}-C .
$$

The proof of (1.16) follows by the same arguments used in 2], adapting the methods in 5 , 12.

The energy estimate (1.15) leads to our second main result which deals with the convergence of $\left\{u_{\varepsilon}\right\}$. Let $\tau: S^{1} \rightarrow \Gamma$ be an orientation preserving $C^{2}$-map satisfying

$$
\left|\tau^{\prime}(s)\right|=\frac{l(\Gamma)}{2 \pi}, \quad \forall s \in S^{1} .
$$

Theorem 2. Assume the same hypotheses as in Theorem 1) Then, there exist a subsequence $\varepsilon_{n} \rightarrow 0$ and $D$ points $a_{1}, \ldots, a_{D}$ in $G$ such that

$$
u_{\varepsilon_{n}} \rightarrow u_{*}=\tau\left(e^{i \phi_{0}} \prod_{j=1}^{D} \frac{z-a_{j}}{\left|z-a_{j}\right|}\right) \text { in } C_{l o c}\left(G \backslash\left\{a_{1}, \ldots, a_{D}\right\}\right),
$$

where $\phi_{0}$ is a smooth harmonic function which is determined by the constraint $u_{*}=\tilde{s}(g)$ on $\partial G$.

The following example demonstrates the importance of using the projection $\tilde{s}$ in Theorem 2 (rather than the usual Euclidean projection).

Example 1. Take $\Gamma=S^{1}$ and fix $a \neq 0$ in the unit disc $B(0,1)$. Set

$$
\Psi(z)=\frac{2}{3}-\left|\frac{z-a}{1-\bar{a} z}\right|+\frac{1}{3} \cdot\left|\frac{z-a}{1-\bar{a} z}\right|^{3},
$$

and define $W$ on $B(0,1)$ by

$$
W(z)=|\nabla \Psi(z)|^{2}=\frac{\left(1-|a|^{2}\right)^{2}}{|1-\bar{a} z|^{4}}\left(1-\left|\frac{z-a}{1-\bar{a} z}\right|^{2}\right)^{2} .
$$

Then complete the definition of $W$ outside $B(0,1)$ in such a way that $W$ will be a $C^{4}$-function on $\mathbb{R}^{2}$ satisfying (1.1)-(1.3). Since $\Psi \in C^{\infty}(B(0,1) \backslash\{a\}$ ), we know, thanks to Proposition 5.1 , that $\Psi$ coincides in $B(0,1) \backslash\{a\}$ with the function defined in (1.9). The level curves of $\Psi$ inside $B(0,1)$ are the circles which are the images, by the Möbius transformation $m_{a}(z)=\frac{z+a}{1+\bar{a} z}$, of the circles centered at 0 ; see Figure 1. Now consider $G=B(0,1)$ and the boundary condition $g\left(e^{i \theta}\right)=b e^{i \theta}$ for some $b \in(0,|a|)$. Then, applying Theorem 2 to this example we see that the degree $D=\operatorname{deg}(\tilde{g})$ is zero since image $(\tilde{s}(g))$ covers only part of $\Gamma=S^{1}$. Hence the limit map $u_{*}=e^{i \phi_{0}}$ is smooth. Note that $\operatorname{deg}(g /|g|)=1$, so using the Euclidean nearest point projection instead of $\tilde{s}$ would lead to a wrong result. 


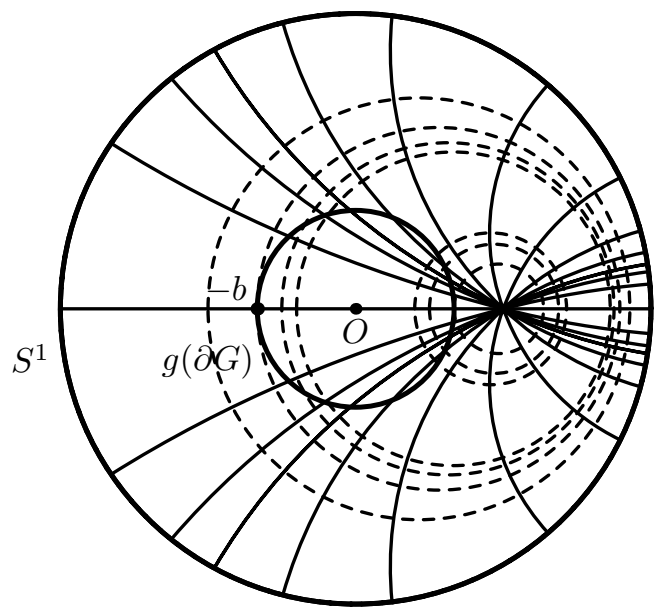

Figure 1. The solid lines are gradient lines of $\Psi$ in the example. The dashed lines are level curves of $\Psi$ (both are circles in our case). The image of $g$ is the circle $B(0, b)$ (heavy solid line).

\section{AN UPPER-BOUND FOR THE ENERGY}

This section is devoted to the proof of the easier part of Theorem 11, namely, the upper-bound. We start by introducing some notation. We first recall some properties of the distance function $\delta$ to $\partial G$ (see [8, Sec. 14.6]). There exists a $b_{0}>0$ such that the distance function $\delta(x)$ to $\partial G$ is $C^{2}$ on $G_{b_{0}}:=\{x \in G: \delta(x) \leq$ $\left.b_{0}\right\}$. Moreover, denoting by $\sigma(x)$ the Euclidean nearest point projection of a point $x \in G_{b_{0}}$ on $\partial G$, we have that the map $x \mapsto(\sigma(x), \delta(x))$ is a $C^{1}$-diffeomorphism of $G_{b_{0}}=\left\{x \in G: \delta(x) \leq b_{0}\right\}$ on $\partial G \times\left[0, b_{0}\right]$. The map $H_{t}(\sigma): \partial G \rightarrow \Sigma_{t}:=\{x \in G:$ $\delta(x)=t\}$ given by $H_{t}(\sigma)=\sigma-t \vec{\nu}(\vec{\nu}$ stands for the exterior normal to $\partial G)$ is also a $C^{1}$-diffeomorphism and its Jacobian satisfies

$$
\left|\mathrm{Jac} H_{t}(\sigma)-1\right| \leq \tilde{c} t, \quad \forall(t, \sigma) \in\left(0, b_{0}\right) \times \partial G .
$$

We shall often identify a point $x \in G_{b_{0}}$ with its $(\sigma, \delta)$-coordinates: $(\sigma(x), \delta(x))$.

We next establish similar notation in the neighborhood of $\Gamma$. We denote by $\tilde{\delta}(x)$ the signed distance function to $\Gamma$, with the convention that $\tilde{\delta}$ is negative inside $\Gamma$ and positive outside. For any $\eta>0$ set

$$
\Gamma_{\eta}=\left\{x \in \mathbb{R}^{2}:|\tilde{\delta}(x)|<\eta\right\} .
$$

Again, by [8, Sec. 14.6], there exists $\eta_{0}>0$ such that $\tilde{\delta} \in C^{2}\left(\Gamma_{\eta_{0}}\right)$, each $x \in \Gamma_{\eta_{0}}$ has a unique nearest point projection $S(x) \in \Gamma$ and the map $S$ is of class $C^{1}$ on $\Gamma_{\eta_{0}}$. Consider an arclength parameterization of $\Gamma$ :

$$
\gamma:[0, l(\Gamma)) \rightarrow \Gamma
$$

For each point $x \in \Gamma_{\eta_{0}}$ we associate a coordinate $\tilde{\sigma}=\tilde{\sigma}(x)$ given by $\tilde{\sigma}(x)=$ $\gamma^{-1}(S(x))$. With the convention that the variable $\tilde{\sigma}$ is taken modulo $l(\Gamma)$ we obtain that the map $x \mapsto(\tilde{\sigma}(x), \tilde{\delta}(x))$ is a local diffeomorphism of class $C^{2}$ of the set $\Gamma_{\eta_{0}}$.

From our assumptions (1.1)-(1.2) it follows that we may write locally in $\Gamma_{\eta_{0}}$, using these coordinates,

$$
W(\tilde{\sigma}, \tilde{\delta})=a(\tilde{\sigma}, \tilde{\delta}) \tilde{\delta}^{2},
$$


for some positive function $a$ of class $C^{2}$. We shall denote

$$
\alpha\left(x_{0}\right)=2 \sqrt{a\left(x_{0}\right)}, \quad \forall x_{0} \in \Gamma .
$$

Note that by (1.10)-(1.11) we also have in $\Omega_{\lambda_{0}}: \Psi(\tilde{\sigma}, \tilde{\delta})=b(\tilde{\sigma}, \tilde{\delta}) \tilde{\delta}^{2}$, for some continuous positive function $b$. Therefore,

$$
\Psi(x) \sim \tilde{\delta}^{2}(x) \sim W(x) \text { in a neighborhood of } \Gamma .
$$

From Proposition 5.1 in the Appendix it follows that there exists a solution $X\left(x_{0}, r\right)$ to the problem

$$
\left\{\begin{array}{l}
\frac{\partial X}{\partial r}=\frac{2 \nabla \Psi(X)}{\alpha\left(x_{0}\right) r}, \\
X\left(x_{0}, 0\right)=x_{0}, \forall x_{0} \in \Gamma,
\end{array}\right.
$$

which is a $C^{1}$-diffeomorphism of a set $\mathcal{N}$ of the form

$$
\mathcal{N}=\left\{(\tilde{s}, r): \tilde{s} \in \Gamma, r \in\left(-r_{1}(\tilde{s}), r_{2}(\tilde{s})\right)\right\},
$$

with $r_{1}(\tilde{s}), r_{2}(\tilde{s})>0, \forall \tilde{s} \in \Gamma$, onto some neighborhood of $\Gamma$ which contains $\Omega_{\lambda_{0}}$, such that $X\left(x_{0}, r\right) \in \Omega$ for $r>0$. In particular, for some $\beta>0$ we have

$$
\beta \leq\left|\frac{\partial X}{\partial \tilde{s}}(\tilde{s}, r)\right| \leq \frac{1}{\beta}, \quad \forall(\tilde{s}, r) \in X^{-1}\left(\Omega_{\lambda_{0}}\right) .
$$

For each $x \in \bar{\Omega}_{\lambda_{0}}$ we can associate a unique number $r(x)$ satisfying $x=X(\tilde{s}(x), r(x))$ $(r(x)>0$ if and only if $x \in \Omega)$.

For $x_{0} \in \Gamma$ let

$$
\gamma_{x_{0}}^{+}:\left(-\infty, t^{+}\left(x_{0}\right)\right] \rightarrow \bar{\Omega}_{\lambda_{0}} \cap \Omega
$$

be the path given by

$$
\gamma_{x_{0}}^{+}(t)=X\left(x_{0}, e^{\alpha t / 2}\right) \quad\left(\text { with } \alpha=\alpha\left(x_{0}\right), \text { see }(\underline{2.5})\right),
$$

with $\Psi\left(\gamma_{x_{0}}^{+}\left(t^{+}\left(x_{0}\right)\right)\right)=\lambda_{0}$. Let $x \in \Omega_{\lambda_{0}} \cap \Omega$ satisfy $\tilde{s}(x)=x_{0}$. Then, $\gamma_{x_{0}}^{+}$satisfies:

$$
\left\{\begin{array}{l}
\dot{\gamma}_{x_{0}}^{+}=\nabla \Psi\left(\gamma_{x_{0}}^{+}\right), \\
\gamma_{x_{0}}^{+}(-\infty)=\tilde{s}(x) \text { and } \gamma_{x_{0}}^{+}\left(\frac{2 \log r(x)}{\alpha}\right)=x .
\end{array}\right.
$$

In a similar manner we define for each $x_{0} \in \Gamma$ a path $\gamma_{x_{0}}^{-}:\left(-\infty, t^{-}\left(x_{0}\right)\right] \rightarrow \bar{\Omega}_{\lambda_{0}} \backslash \Omega$ by $\gamma_{x_{0}}^{-}(t)=X\left(x_{0},-e^{\alpha t / 2}\right)$ which satisfies

$$
\left\{\begin{array}{l}
\dot{\gamma}_{x_{0}}^{-}=\nabla \Psi\left(\gamma_{x_{0}}^{-}\right), \\
\gamma_{x_{0}}^{-}(-\infty)=\tilde{s}(x) \text { and } \gamma_{x_{0}}^{-}\left(\frac{2 \log (-r(x))}{\alpha}\right)=x,
\end{array}\right.
$$

for every $x \in \bar{\Omega}_{\lambda_{0}} \backslash \Omega$ such that $\tilde{s}(x)=x_{0}$. It will be convenient to also set $\gamma_{x_{0}}^{0}(t) \equiv x_{0}, \forall x_{0} \in \Gamma, \forall t$.

We are now ready to state and prove the upper-bound part of Theorem 1.

Proposition 2.1. Under the hypotheses of Theorem 1 we have

$$
E_{\varepsilon}\left(u_{\varepsilon}\right) \leq \frac{2}{\varepsilon} \int_{\partial G} \Psi(g(\sigma)) d \sigma+D \frac{l^{2}(\Gamma)}{2 \pi} \log \frac{1}{\varepsilon}+C, \forall \varepsilon>0 .
$$

Proof. Clearly it is enough to construct $\left\{v_{\varepsilon}\right\} \subset H_{g}^{1}\left(G, \mathbb{R}^{2}\right)$ such that,

$$
E_{\varepsilon}\left(v_{\varepsilon}\right) \leq \frac{2}{\varepsilon} \int_{\partial G} \Psi(g(s)) d s+D \frac{l^{2}(\Gamma)}{2 \pi} \log \frac{1}{\varepsilon}+C .
$$


Denoting $S(\sigma)=\operatorname{sgn}(r(g(\sigma)))$ for $\sigma \in \partial G$, we define $v_{\varepsilon}$ for $\varepsilon$ small by:

$$
v_{\varepsilon}(\sigma, \delta)= \begin{cases}\gamma_{\tilde{s}(g(\sigma))}^{S(\sigma)}\left(\frac{2 \log |r(g(\sigma))|}{\alpha}-\frac{\delta}{\varepsilon}\right), & \delta \leq \varepsilon^{1 / 2}, \\ \frac{2 \varepsilon^{1 / 2}-\delta}{\varepsilon^{1 / 2}} v_{\varepsilon}\left(\sigma, \varepsilon^{1 / 2}\right)+\frac{\delta-\varepsilon^{1 / 2}}{\varepsilon^{1 / 2}} \tilde{s}(g(\sigma)), & \varepsilon^{1 / 2}<\delta \leq 2 \varepsilon^{1 / 2}, \\ \tilde{s}(g(\sigma)), & 2 \varepsilon^{1 / 2}<\delta \leq b_{0} .\end{cases}
$$

It remains to define $v_{\varepsilon}$ on $G \backslash G_{b_{0}}$. We choose $D$ points $a_{1}, \ldots, a_{D} \in G \backslash G_{b_{0}}$ and then $r_{0}$ such that

$$
0<r_{0} \leq \frac{1}{2} \min \left\{\min _{i \neq j}\left|a_{i}-a_{j}\right|, \min _{i} \delta\left(a_{i}\right)-b_{0}\right\} .
$$

On $\left(G \backslash G_{b_{0}}\right) \backslash \bigcup_{j=1}^{D} B\left(a_{j}, r_{0}\right)$ we set $v_{\varepsilon}=F_{0}$ where $F_{0}$ is any $\Gamma$-valued $C^{1}$-map satisfying:

$$
F_{0}(x)= \begin{cases}\tilde{s}(g(\sigma(x))) & \text { on } \Sigma_{b_{0}}=\partial\left(G \backslash G_{b_{0}}\right), \\ \tau\left(\frac{x-a_{j}}{\left|x-a_{j}\right|}\right) & \text { on } \partial B\left(a_{j}, r_{0}\right), j=1, \ldots, D(\text { see (1.17) }) .\end{cases}
$$

Finally, on each $B\left(a_{j}, r_{0}\right)$ we define $v_{\varepsilon}(x)=f_{\varepsilon}\left(\left|x-a_{j}\right|\right) \cdot \tau\left(\frac{x-a_{j}}{\left|x-a_{j}\right|}\right)$ where the scalar function $f_{\varepsilon}$ is defined by:

$$
f_{\varepsilon}(r)= \begin{cases}1 & \text { for } \varepsilon<r \leq r_{0} \\ \frac{r}{\varepsilon} & \text { for } 0 \leq r \leq \varepsilon\end{cases}
$$

It is easy to verify that

$$
E_{\varepsilon}\left(v_{\varepsilon}, B\left(a_{j}, r_{0}\right)\right)=\frac{l^{2}(\Gamma)}{2 \pi} \log \frac{1}{\varepsilon}+C, \quad j=1, . ., D,
$$

which implies that

$$
E_{\varepsilon}\left(v_{\varepsilon}, G \backslash G_{b_{0}}\right) \leq D \frac{l^{2}(\Gamma)}{2 \pi} \log \frac{1}{\varepsilon}+C .
$$

It remains to estimate $E_{\varepsilon}\left(v_{\varepsilon}, G_{b_{0}}\right)$. Since $X$ is a $C^{1}$-solution of (2.7), it follows that $\gamma_{x_{0}}^{ \pm}$satisfy

$$
\left|\gamma_{x_{0}}^{ \pm}(t)-x_{0}\right|,\left|\dot{\gamma}_{x_{0}}^{ \pm}(t)\right| \leq C e^{c t} \text { and } \Psi\left(\gamma_{x_{0}}^{ \pm}(t)\right) \leq C e^{c t}, \forall x_{0} \in \Gamma
$$

for some positive constants $c, C$. Using these estimates we conclude easily that

$$
E_{\varepsilon}\left(v_{\varepsilon}, G_{b_{0}} \backslash G_{\varepsilon^{1 / 2}}\right) \leq C \text {. }
$$

Finally, on $G_{\varepsilon^{1 / 2}}$ we have

$$
\frac{\partial v_{\varepsilon}}{\partial \delta}(\sigma, \delta)=-\frac{1}{\varepsilon} \cdot \dot{\gamma}_{\tilde{s}(g(\sigma))}\left(\frac{2 \log r(g(\sigma))}{\alpha}-\frac{\delta}{\varepsilon}\right)=-\frac{1}{\varepsilon} \cdot \nabla \Psi\left(v_{\varepsilon}(\sigma, \delta)\right) .
$$

Since by the construction of $v_{\varepsilon},-\frac{\partial \Psi\left(v_{\varepsilon}\right)}{\partial \delta} \geq 0$, it follows from (2.10)-(2.11), (1.10) and (2.1) that

$$
\begin{aligned}
I_{1}: & =\int_{G_{\varepsilon^{1 / 2}}}\left|\frac{\partial v_{\varepsilon}}{\partial \delta}\right|^{2}+\frac{W\left(v_{\varepsilon}\right)}{\varepsilon^{2}}=-\frac{2}{\varepsilon} \int_{G_{\varepsilon^{1 / 2}}} \nabla \Psi\left(v_{\varepsilon}\right) \frac{\partial v_{\varepsilon}}{\partial \delta} \\
& =\frac{2}{\varepsilon} \int_{G_{\varepsilon^{1 / 2}}}-\frac{\partial\left(\Psi\left(v_{\varepsilon}\right)\right)}{\partial \delta} \leq \frac{2}{\varepsilon} \int_{\partial G} \int_{0}^{\varepsilon^{1 / 2}}-\frac{\partial\left(\Psi\left(v_{\varepsilon}\right)\right)}{\partial \delta}(1+\tilde{c} \delta) d \delta d \sigma .
\end{aligned}
$$


Next, for each $\sigma \in \partial G$ we have

$$
\begin{aligned}
& \int_{0}^{\varepsilon^{1 / 2}}-\frac{\partial\left(\Psi\left(v_{\varepsilon}(\sigma, \delta)\right)\right)}{\partial \delta}(1+\tilde{c} \delta) d \delta \\
& =\Psi\left(v_{\varepsilon}(\sigma, 0)\right)-\Psi\left(v_{\varepsilon}\left(\sigma, \varepsilon^{1 / 2}\right)\right)\left(1+\tilde{c} \varepsilon^{1 / 2}\right)+\int_{0}^{\varepsilon^{1 / 2}} \tilde{c} \Psi\left(v_{\varepsilon}(\sigma, \delta)\right) d \delta \leq C,
\end{aligned}
$$

where $C$ is independent of $\sigma$ and $\varepsilon$. An immediate consequence of (2.16) is that $I_{1} \leq \frac{C}{\varepsilon}$. In particular, $\int_{G_{\varepsilon^{1 / 2}}} W\left(v_{\varepsilon}\right) \leq C \varepsilon$ and using (2.6) we obtain that

$$
\int_{G_{\varepsilon^{1 / 2}}} \Psi\left(v_{\varepsilon}\right) \leq C \varepsilon
$$

Integrating (2.16) over $\sigma \in \partial G$ and using (2.17) yields

$$
I_{1} \leq \frac{2}{\varepsilon} \int_{\partial G} \Psi(g(\sigma)) d \sigma+O(1) .
$$

It is easy to verify that $\left|\frac{\partial v_{\varepsilon}}{\partial \sigma}\right| \leq C$ and therefore

$$
I_{2}:=\int_{G_{\varepsilon^{1 / 2}}}\left|\frac{\partial v_{\varepsilon}}{\partial \sigma}\right|^{2} \leq C .
$$

The result follows by combining (2.18) $-(2.19)$ with (2.13) $-(2.14)$.

\section{A LOWER-BOUND OF THE ENERGY}

In this section we shall prove the lower-bound part of Theorem 1 . We begin with a simple lemma which provides two basic estimates satisfied by the minimizer $u_{\varepsilon}$. The proofs are very similar to those of the analogous results for the GinzburgLandau functional (see [4]).

Lemma 3.1. There exist two positive constants $\widetilde{C}_{1}$ and $\widetilde{C}_{2}$ such that for every $\varepsilon$ we have

$$
\left|u_{\varepsilon}\right| \leq \widetilde{C}_{1} \text { in } G
$$

and

$$
\left\|\nabla u_{\varepsilon}\right\|_{L^{\infty}(G)} \leq \frac{\widetilde{C}_{2}}{\varepsilon} .
$$

Proof. We claim that (3.1) holds with

$$
\widetilde{C}_{1}=\max \left(\max _{x \in \partial G}|g(x)|, R_{0}\right) \quad(\text { see }(1.3)) .
$$

Indeed, assuming by negation that (3.1) does not hold, we easily get from (1.3) that the function

$$
\tilde{u}_{\varepsilon}(x)= \begin{cases}u_{\varepsilon}(x) & \text { if }\left|u_{\varepsilon}(x)\right| \leq \widetilde{C}_{1}, \\ \widetilde{C}_{1} \frac{u_{\varepsilon}(x)}{\left|u_{\varepsilon}(x)\right|} & \text { if }\left|u_{\varepsilon}(x)\right|>\widetilde{C}_{1},\end{cases}
$$

belongs to $H_{g}^{1}\left(G, \mathbb{R}^{2}\right)$ and satisfies $E_{\varepsilon}\left(\tilde{u}_{\varepsilon}\right)<E_{\varepsilon}\left(u_{\varepsilon}\right)$, contradicting the minimizing property of $u_{\varepsilon}$.

The Euler-Lagrange equation satisfied by $u_{\varepsilon}$ is

$$
\Delta u_{\varepsilon}=\left(\frac{1}{2 \varepsilon^{2}}\right) \nabla W\left(u_{\varepsilon}\right) .
$$


Applying standard elliptic estimates for the rescaled function $v_{\varepsilon}(y)=u_{\varepsilon}(\varepsilon y)$ (like in [4, 12]) yields the $L^{\infty}$-bound for the gradient.

The main result of this section is the following

Proposition 3.1. There exists a constant $C>0$ such that,

$$
E_{\varepsilon}\left(u_{\varepsilon}\right) \geq \frac{2}{\varepsilon} \int_{\partial G} \Psi(g(\sigma)) d \sigma+D \frac{l^{2}(\Gamma)}{2 \pi} \log \frac{1}{\varepsilon}-C, \forall \varepsilon \in(0,1) .
$$

The proof relies on several lemmas.

Lemma 3.2. Let I be a compact subinterval of $(1 / 2,1)$. Then, there exist constants $a=a(I) \in(0,1)$ and $\mu=\mu(I), C=C(I)>0$ and, for any $\alpha \in I$ and $\varepsilon \in(0,1), a$ set $J=J_{\alpha, \varepsilon} \subset(0, C(I))$ satisfying meas $(J) \geq \mu$, such that

$$
\int_{G_{c_{0} \varepsilon^{\alpha}}}\left|\nabla u_{\varepsilon}\right|^{2}+\frac{W\left(u_{\varepsilon}\right)}{\varepsilon^{2}} \geq \frac{2}{\varepsilon} \int_{\partial G} \Psi(g(\sigma)) d \sigma-C
$$

and

$$
\int_{\Sigma_{c_{0} \varepsilon^{\alpha}}} \Psi\left(u_{\varepsilon}(\sigma)\right) d \sigma \leq C \varepsilon^{1+a}
$$

for all $c_{0} \in J$.

Proof. For $\alpha \in I$ and any $c>0$ we have by the Cauchy-Schwarz inequality

$$
\begin{aligned}
\int_{G_{c \varepsilon} \alpha}\left|\nabla u_{\varepsilon}\right|^{2}+\frac{W\left(u_{\varepsilon}\right)}{\varepsilon^{2}} & \geq \frac{2}{\varepsilon} \int_{G_{c \varepsilon} \alpha}\left|\nabla u_{\varepsilon}\right|\left(W\left(u_{\varepsilon}\right)\right)^{1 / 2} \\
& \geq \frac{2}{\varepsilon} \int_{G_{c \varepsilon} \alpha}\left|\nabla\left(\Psi\left(u_{\varepsilon}\right)\right)\right| \geq \frac{2}{\varepsilon} \int_{G_{c \varepsilon} \alpha} \nabla\left(\Psi\left(u_{\varepsilon}\right)\right) \cdot V,
\end{aligned}
$$

for every $C^{1}$ vector field $V$ such that $|V| \leq 1$ on $G_{c \varepsilon^{\alpha}}$. Choosing $V=-\nabla \delta$ yields,

$$
\begin{aligned}
& \int_{G_{c \varepsilon} \alpha}\left|\nabla u_{\varepsilon}\right|^{2}+\frac{W\left(u_{\varepsilon}\right)}{\varepsilon^{2}} \\
& \quad \geq \frac{2}{\varepsilon} \int_{\partial G} \Psi(g(\sigma)) d \sigma-\frac{2}{\varepsilon} \int_{\Sigma_{c \varepsilon} \alpha} \Psi\left(u_{\varepsilon}(\sigma)\right) d \sigma-\frac{2}{\varepsilon} \int_{G_{c \varepsilon} \alpha} \Psi\left(u_{\varepsilon}\right) \operatorname{div} V \\
& \quad:=I_{1}+I_{2}+I_{3} .
\end{aligned}
$$

By the upper-bound (2.12) we know that $\int_{G} W\left(u_{\varepsilon}\right) \leq C \varepsilon$, so that by (3.1) and (2.6) we deduce that $W\left(u_{\varepsilon}\right) \sim \Psi\left(u_{\varepsilon}\right)$ and therefore $I_{3}$ is bounded (uniformly in $\varepsilon$ ).

Using $\int_{G} W\left(u_{\varepsilon}\right) \leq C \varepsilon$ again we obtain that $\int_{G} \Psi\left(u_{\varepsilon}\right) \leq C \varepsilon$ and we deduce the existence of $c_{1} \in(0,1)$ such that

$$
\int_{\Sigma_{c_{1} \varepsilon^{\alpha}}} \Psi\left(u_{\varepsilon}(\sigma)\right) d \sigma \leq C \varepsilon^{1-\alpha} .
$$

For $c=c_{1}$ we get $I_{2} \leq C \varepsilon^{-\alpha}$, and (3.7) now reads:

$$
\int_{G_{c_{1} \varepsilon^{\alpha}}}\left|\nabla u_{\varepsilon}\right|^{2}+\frac{W\left(u_{\varepsilon}\right)}{\varepsilon^{2}} \geq \frac{2}{\varepsilon} \int_{\partial G} \Psi(g(\sigma)) d \sigma-C \varepsilon^{-\alpha} .
$$

Using the upper-bound again we obtain that

$$
\int_{G \backslash G_{c_{1} \varepsilon^{\alpha}}}\left|\nabla u_{\varepsilon}\right|^{2}+\frac{W\left(u_{\varepsilon}\right)}{\varepsilon^{2}} \leq C \varepsilon^{-\alpha} .
$$


In particular, $\int_{G \backslash G_{c_{1} \varepsilon^{\alpha}}} W\left(u_{\varepsilon}\right) \leq C \varepsilon^{2-\alpha}$ and then there exists $c_{2} \in(1,2)$ such that $\int_{\Sigma_{c_{2} \varepsilon^{\alpha}}} W\left(u_{\varepsilon}(\sigma)\right) d \sigma \leq C \varepsilon^{2-2 \alpha}$, and therefore also

$$
\int_{\Sigma_{c_{2} \varepsilon^{\alpha}}} \Psi\left(u_{\varepsilon}(\sigma)\right) d \sigma \leq C \varepsilon^{2-2 \alpha}
$$

This last estimate is then plugged back in (3.7) and the argument is repeated.

Let $n=n(\alpha)$ be such that

$$
\frac{n-1}{n} \leq \alpha<\frac{n}{n+1} .
$$

Clearly, $\sup \{n(\alpha)$ satisfying (3.8) $: \alpha \in I\}<\infty$. Applying the above argument $n$ times we obtain the existence of some $c_{n} \in(n-1, n)$ such that

$$
\int_{G_{c_{n} \varepsilon^{\alpha}}}\left|\nabla u_{\varepsilon}\right|^{2}+\frac{W\left(u_{\varepsilon}\right)}{\varepsilon^{2}} \geq \frac{2}{\varepsilon} \int_{\partial G} \Psi(g(\sigma)) d \sigma-C \varepsilon^{n-1-n \alpha} .
$$

Using the upper-bound once more we get that

$$
\int_{G \backslash G_{c_{n} \varepsilon^{\alpha}}}\left|\nabla u_{\varepsilon}\right|^{2}+\frac{W\left(u_{\varepsilon}\right)}{\varepsilon^{2}} \leq C\left(\varepsilon^{n-1-n \alpha}+|\log \varepsilon|\right),
$$

which leads to

$$
\int_{G \backslash G_{c_{n} \varepsilon^{\alpha}}} \Psi\left(u_{\varepsilon}\right) \leq C \varepsilon^{2}\left(\varepsilon^{n-1-n \alpha}+|\log \varepsilon|\right),
$$

and to the existence of a $c_{0} \in(n, n+1)$ such that

$$
\int_{\Sigma_{c_{0} \varepsilon^{\alpha}}} \Psi\left(u_{\varepsilon}(\sigma)\right) d \sigma \leq C \varepsilon^{(n+1)(1-\alpha)}+C \varepsilon^{2-\alpha}|\log \varepsilon| \leq C \varepsilon^{1+a},
$$

for any $a$ satisfying

$$
0<a<\min (1-\alpha,(n+1)(1-\alpha)-1) .
$$

Clearly, an $a(I)$ satisfying (3.10) for all $\alpha \in I$ can be chosen. Furthermore, the measure of the set of $c_{0}$ 's in $(n, n+1)$ satisfying (3.9) is bounded from below, uniformly in $\alpha \in I \subset \subset(1 / 2,1)$ and $\varepsilon \in(0,1)$, by some positive $\mu$. We therefore proved (3.6), and using (3.9) in (3.7) with $c=c_{0}$ yields that $I_{2} \leq C$, and (3.5) follows as well.

The next lemma provides a simple pointwise lower-bound for $\left|\nabla u_{\varepsilon}\right|$. We denote

$$
G_{0}^{\varepsilon}:=\left\{x \in G: u_{\varepsilon}(x) \in \Omega_{\lambda_{0}}\right\} .
$$

Lemma 3.3. We have

$$
\left|\nabla u_{\varepsilon}\right|^{2} \geq \frac{\left|\nabla\left(\Psi\left(u_{\varepsilon}\right)\right)\right|^{2}}{W\left(u_{\varepsilon}\right)} \quad \text { a.e. in } G
$$

and

$$
\left|\nabla u_{\varepsilon}\right|^{2} \geq \beta\left|\nabla\left(\tilde{s}\left(u_{\varepsilon}\right)\right)\right|^{2}+\frac{\left|\nabla\left(\Psi\left(u_{\varepsilon}\right)\right)\right|^{2}}{W\left(u_{\varepsilon}\right)} \quad \text { in } G_{0}^{\varepsilon},
$$

for some $\beta>0$, independent of $\varepsilon$. 
Proof. The estimate (3.12) follows immediately from the inequality

$$
\left|\nabla\left(\Psi\left(u_{\varepsilon}\right)\right)\right|^{2}=\left|\nabla \Psi\left(u_{\varepsilon}\right) \cdot \nabla u_{\varepsilon}\right|^{2} \leq\left|\nabla \Psi\left(u_{\varepsilon}\right)\right|^{2}\left|\nabla u_{\varepsilon}\right|^{2}=W\left(u_{\varepsilon}\right)\left|\nabla u_{\varepsilon}\right|^{2} \text {, a.e. in } G \text {. }
$$

Next, at each point $y \in \Omega_{\lambda_{0}}$ we denote by $\vec{\nu}=\vec{\nu}(y)$ a unit vector in the direction of $\nabla \Psi(y)$ and by $\vec{\tau}=\vec{\tau}(y)$ an orthogonal unit vector, in the direction of $\nabla \tilde{s}(y)$. For $x \in G_{0}^{\varepsilon}$ we may then write

$$
\left|\nabla u_{\varepsilon}\right|^{2}=\left|\nabla_{\nu} u_{\varepsilon}\right|^{2}+\left|\nabla_{\tau} u_{\varepsilon}\right|^{2}
$$

and get a more precise form of (3.14) in this case:

$$
\left|\nabla_{\nu} u_{\varepsilon}\right|^{2}=\frac{\left|\nabla\left(\Psi\left(u_{\varepsilon}\right)\right)\right|^{2}}{W\left(u_{\varepsilon}\right)}
$$

Finally, since

$$
\nabla_{\tau} u_{\varepsilon}=\nabla u_{\varepsilon} \cdot \frac{\nabla \tilde{s}\left(u_{\varepsilon}\right)}{\left|\nabla \tilde{s}\left(u_{\varepsilon}\right)\right|}=\frac{\nabla\left(\tilde{s}\left(u_{\varepsilon}\right)\right)}{\left|\nabla \tilde{s}\left(u_{\varepsilon}\right)\right|}
$$

and $|\nabla \tilde{s}(y)| \leq \frac{1}{\sqrt{\beta}}, \forall y \in \Omega_{\lambda_{0}}$ for some $\beta>0$, we conclude that

$$
\left|\nabla_{\tau} u_{\varepsilon}\right|^{2} \geq \beta\left|\nabla\left(\tilde{s}\left(u_{\varepsilon}\right)\right)\right|^{2},
$$

as required.

In the sequel we shall fix an interval $I \subset \subset(1 / 2,1)$ and consider $\alpha \in I$. An important role in the proof of Proposition 3.1 is played by the scalar function $d_{0 \varepsilon}$ which is defined as the minimizer for the problem

$$
\min \left\{\int_{G_{c_{0} \varepsilon^{\alpha}}} \frac{|\nabla d|^{2}}{W\left(u_{\varepsilon}\right)+\varepsilon^{2}}: d \in H^{1}\left(G_{c_{0} \varepsilon^{\alpha}}\right), d=\Psi\left(u_{\varepsilon}\right) \text { on } \partial G_{c_{0} \varepsilon^{\alpha}}\right\} .
$$

The existence and uniqueness of $d_{0 \varepsilon}$ is standard. The Euler-Lagrange equation satisfied by $d_{0 \varepsilon}$ is

$$
\left\{\begin{array}{l}
\operatorname{div}\left(\frac{\nabla d_{0 \varepsilon}}{W\left(u_{\varepsilon}\right)+\varepsilon^{2}}\right)=0 \text { in } G_{c_{0} \varepsilon^{\alpha}} \\
d_{0 \varepsilon}=\Psi\left(u_{\varepsilon}\right) \text { on } \partial G_{c_{0} \varepsilon^{\alpha}}
\end{array}\right.
$$

for any $c_{0} \in J$ (see Lemma 3.2).

Lemma 3.4. There exists $\varepsilon_{0}>0$ such that for every $\varepsilon \leq \varepsilon_{0}$ and $\alpha \in I$ we have:

$$
\left.0 \leq d_{0 \varepsilon} \leq \lambda_{1} \quad \text { in } G_{c_{0} \varepsilon^{\alpha}} \quad \text { (see (1.14) }\right) .
$$

Proof. It is enough to show that

$$
d_{0 \varepsilon}=\Psi\left(u_{\varepsilon}\right) \leq \lambda_{1} \text { on } \partial G_{c_{0} \varepsilon^{\alpha}} \text { (for } \varepsilon \text { small), }
$$

and then apply the maximum principle to (3.18). The inequality on $\partial G$ is clear from (1.14). As for the bound on $\Sigma_{c_{0} \varepsilon^{\alpha}}=\left\{x \in G: \delta(x)=c_{0} \varepsilon^{\alpha}\right\}$, note first that by (3.6) and (2.6) we have

$$
\int_{\Sigma_{c_{0} \varepsilon^{\alpha}}} W\left(u_{\varepsilon}\right) \leq C \varepsilon^{1+a}
$$

Let $x_{0} \in \Sigma_{c_{0} \varepsilon^{\alpha}}$ satisfy

$$
\left|\tilde{\delta}\left(u_{\varepsilon}\left(x_{0}\right)\right)\right|=m:=\max _{\Sigma_{c_{0} \varepsilon^{\alpha}}}\left|\tilde{\delta}\left(u_{\varepsilon}(x)\right)\right| .
$$


By (3.2) we have:

$$
\left|\tilde{\delta}\left(u_{\varepsilon}(x)\right)\right| \geq m / 2 \text { for every } x \in \Sigma_{c_{0} \varepsilon^{\alpha}} \text { s.t. }\left|x_{0}-x\right| \leq \frac{m \varepsilon}{2 c},
$$

for some $c>0$. For such $x$ we obtain, for some $a_{0}>0$ (see (2.4)):

$$
W\left(u_{\varepsilon}(x)\right) \geq a_{0} \tilde{\delta}^{2}\left(u_{\varepsilon}(x)\right) \geq \frac{a_{0} m^{2}}{4},
$$

so that, for $\varepsilon$ small,

$$
a_{0} \frac{m^{3} \varepsilon}{8 c}=\frac{m \varepsilon}{2 c} \cdot \frac{a_{0} m^{2}}{4} \leq \int_{\Sigma_{c_{0} \varepsilon^{\alpha}}} W\left(u_{\varepsilon}\right) \leq C \varepsilon^{1+a},
$$

which leads to $m \leq \varepsilon^{b}$ for some $b>0$. Therefore, also $\Psi\left(u_{\varepsilon}\right) \leq C W\left(u_{\varepsilon}\right) \leq C \varepsilon^{2 b}<$ $\lambda_{1}$ on $\Sigma_{c_{0} \varepsilon^{\alpha}}$, for $\varepsilon$ small enough, and (3.20) follows.

By the definition of $d_{0 \varepsilon}$, Lemma 3.3 and the upper-bound (2.12) we deduce that

$$
\int_{G_{c_{0} \varepsilon^{\alpha}}} \frac{\left|\nabla d_{0 \varepsilon}\right|^{2}}{W\left(u_{\varepsilon}\right)+\varepsilon^{2}} \leq \int_{G_{c_{0} \varepsilon^{\alpha}}} \frac{\left|\nabla\left(\Psi\left(u_{\varepsilon}\right)\right)\right|^{2}}{W\left(u_{\varepsilon}\right)+\varepsilon^{2}} \leq \frac{C}{\varepsilon} .
$$

Put $d_{1 \varepsilon}=\Psi\left(u_{\varepsilon}\right)-d_{0 \varepsilon}$. Then,

$$
\frac{\left|\nabla\left(\Psi\left(u_{\varepsilon}\right)\right)\right|^{2}}{W\left(u_{\varepsilon}\right)+\varepsilon^{2}}=\frac{1}{W\left(u_{\varepsilon}\right)+\varepsilon^{2}}\left(\left|\nabla d_{0 \varepsilon}\right|^{2}+2 \nabla d_{0 \varepsilon} \cdot \nabla d_{1 \varepsilon}+\left|\nabla d_{1 \varepsilon}\right|^{2}\right) .
$$

The motivation for introducing $d_{1 \varepsilon}$ is the following simple consequence of (3.22), (3.18) and Green's formula:

$$
\int_{G_{c_{0} \varepsilon^{\alpha}}} \frac{\left|\nabla\left(\Psi\left(u_{\varepsilon}\right)\right)\right|^{2}}{W\left(u_{\varepsilon}\right)+\varepsilon^{2}}=\int_{G_{c_{0} \varepsilon^{\alpha}}} \frac{\left|\nabla d_{0 \varepsilon}\right|^{2}}{W\left(u_{\varepsilon}\right)+\varepsilon^{2}}+\frac{\left|\nabla d_{1 \varepsilon}\right|^{2}}{W\left(u_{\varepsilon}\right)+\varepsilon^{2}} .
$$

In fact, from (3.16) and (3.23) we conclude that

$$
\int_{G_{c_{0} \varepsilon^{\alpha}}}\left|\nabla_{\nu} u_{\varepsilon}\right|^{2} \geq \int_{G_{c_{0} \varepsilon^{\alpha}}} \frac{\left|\nabla d_{0 \varepsilon}\right|^{2}}{W\left(u_{\varepsilon}\right)+\varepsilon^{2}}+\frac{\left|\nabla d_{1 \varepsilon}\right|^{2}}{W\left(u_{\varepsilon}\right)+\varepsilon^{2}} .
$$

The next lemma provides a crucial lower-bound for the first term on the r.h.s. of (3.24).

Lemma 3.5. There exists a constant $C_{1}=C_{1}(I)$ such that

$$
\int_{G_{c_{0} \varepsilon^{\alpha}}} \frac{\left|\nabla d_{0 \varepsilon}\right|^{2}}{W\left(u_{\varepsilon}\right)+\varepsilon^{2}}+\frac{W\left(u_{\varepsilon}\right)+\varepsilon^{2}}{\varepsilon^{2}} \geq \frac{2}{\varepsilon} \int_{\partial G} \Psi(g(\sigma)) d \sigma-C_{1}, \forall \varepsilon \in(0,1), \forall \alpha \in I .
$$

Proof. As in the proof of Lemma 3.2 we have for $V=-\nabla \delta$ :

$$
\begin{aligned}
\int_{G_{c_{0} \varepsilon^{\alpha}} \frac{\left|\nabla d_{0 \varepsilon}\right|^{2}}{W\left(u_{\varepsilon}\right)+\varepsilon^{2}}+\frac{W\left(u_{\varepsilon}\right)+\varepsilon^{2}}{\varepsilon^{2}}} & \geq \frac{2}{\varepsilon} \int_{G_{c_{0} \varepsilon^{\alpha}}}\left|\nabla d_{0 \varepsilon}\right| \geq \frac{2}{\varepsilon} \int_{G_{c_{0} \varepsilon^{\alpha}}} \nabla d_{0 \varepsilon} \cdot V \\
& =\frac{2}{\varepsilon} \int_{\partial G_{c_{0} \varepsilon^{\alpha}}} \Psi\left(u_{\varepsilon}(\sigma)\right) d \sigma-\frac{2}{\varepsilon} \int_{G_{c_{0} \varepsilon^{\alpha}}} d_{0 \varepsilon} \operatorname{div} V \\
& \geq \frac{2}{\varepsilon} \int_{\partial G} \Psi(g(\sigma)) d \sigma-\frac{2}{\varepsilon} \int_{G_{c_{0} \varepsilon^{\alpha}}} d_{0 \varepsilon} \operatorname{div} V-C,
\end{aligned}
$$


where in the last inequality we used (3.6). Therefore, in order to conclude we only need to prove that

$$
\int_{G_{c_{0} \varepsilon^{\alpha}}} d_{0 \varepsilon} \leq C \varepsilon
$$

Fix any $\delta \in\left(0, c_{0} \varepsilon^{\alpha}\right)$ and let $\delta_{0}=\delta / 5$. By the upper-bound (2.12) there exists $\delta_{1} \in\left(\delta_{0}, 2 \delta_{0}\right)$ such that

$$
\int_{\Sigma_{\delta_{1}}} W\left(u_{\varepsilon}\right) \leq \frac{C \varepsilon}{\delta}
$$

By the same argument as in the proof of Lemma 3.2 we get that

$$
\int_{G \backslash G_{\delta_{1}}} W\left(u_{\varepsilon}\right) \leq \frac{C \varepsilon^{2}}{\delta}+C \varepsilon^{2}|\log \varepsilon|
$$

Repeating the argument we find $\delta_{2} \in\left(2 \delta_{0}, 3 \delta_{0}\right)$ such that

$$
\int_{G \backslash G \delta_{2}} W\left(u_{\varepsilon}\right) \leq \frac{C \varepsilon^{3}}{\delta^{2}}+C \varepsilon^{2}|\log \varepsilon| .
$$

Repeating the argument one last time we deduce that there exists $\delta_{3} \in\left(3 \delta_{0}, 4 \delta_{0}\right)$ such that

$$
\int_{G \backslash G_{\delta_{3}}} W\left(u_{\varepsilon}\right) \leq \frac{C \varepsilon^{4}}{\delta^{3}}+C \varepsilon^{2}|\log \varepsilon| .
$$

Hence, for any $\delta \in\left(\varepsilon, c_{0} \varepsilon^{\alpha}\right)$ we have

$$
\int_{G \backslash G_{\delta}} W\left(u_{\varepsilon}\right) \leq \frac{C \varepsilon^{4}}{\delta^{3}}+C \varepsilon^{2}|\log \varepsilon| .
$$

Next, using (2.1) and (3.6) we obtain

$$
\begin{aligned}
\int_{G_{c_{0} \varepsilon^{\alpha} \backslash G_{\varepsilon}}} d_{0 \varepsilon} & \leq C \int_{\varepsilon}^{c_{0} \varepsilon^{\alpha}}\left(\int_{\partial G} d_{0 \varepsilon}(\sigma, \delta) d \sigma\right) d \delta \\
& \leq C \int_{\varepsilon}^{c_{0} \varepsilon^{\alpha}}\left(\int_{\partial G}\left(d_{0 \varepsilon}\left(\sigma, c_{0} \varepsilon^{\alpha}\right)+\int_{\delta}^{c_{0} \varepsilon^{\alpha}}\left|\nabla d_{0 \varepsilon}(\sigma, t)\right| d t\right) d \sigma\right) d \delta \\
& \leq C \int_{\varepsilon}^{c_{0} \varepsilon^{\alpha}}\left(\int_{G \backslash G_{\delta}}\left|\nabla d_{0 \varepsilon}\right|\right) d \delta+C \varepsilon^{1+a+\alpha} .
\end{aligned}
$$

By the Cauchy-Schwarz inequality, (3.21) and (3.27) we get:

$$
\begin{aligned}
\int_{\varepsilon}^{c_{0} \varepsilon^{\alpha}} & \left(\int_{G \backslash G_{\delta}}\left|\nabla d_{0 \varepsilon}\right|\right) d \delta \\
\leq & \int_{\varepsilon}^{c_{0} \varepsilon^{\alpha}}\left(\int_{G \backslash G_{\delta}} \frac{\left|\nabla d_{0 \varepsilon}\right|^{2}}{W\left(u_{\varepsilon}\right)+\varepsilon^{2}}\right)^{1 / 2}\left(\int_{G \backslash G_{\delta}} W\left(u_{\varepsilon}\right)+\varepsilon^{2}\right)^{1 / 2} d \delta \\
\leq & \frac{C}{\varepsilon^{1 / 2}} \int_{\varepsilon}^{c_{0} \varepsilon^{\alpha}}\left(\int_{G \backslash G_{\delta}} W\left(u_{\varepsilon}\right)+\varepsilon^{2}\right)^{1 / 2} d \delta \\
\leq & \frac{C}{\varepsilon^{1 / 2}} \int_{\varepsilon}^{c_{0} \varepsilon^{\alpha}}\left(\frac{\varepsilon^{2}}{\delta^{3 / 2}}+\varepsilon|\log \varepsilon|^{1 / 2}+\varepsilon\right) d \delta \\
\leq & \left.-C \varepsilon^{3 / 2} \cdot s^{-1 / 2}\right]_{\varepsilon}^{c_{0} \varepsilon^{\alpha}}+c \varepsilon^{\alpha+1 / 2}|\log \varepsilon|^{1 / 2}+C \varepsilon^{1 / 2+\alpha} \leq C \varepsilon .
\end{aligned}
$$


Combining (3.29) with (3.28) we obtain that

$$
\int_{G_{c_{0} \varepsilon^{\alpha} \backslash G_{\varepsilon}}} d_{0 \varepsilon} \leq C \varepsilon
$$

On the other hand, the inequality

$$
\int_{G_{\varepsilon}} d_{0 \varepsilon} \leq C \varepsilon
$$

is obvious since $\left|G_{\varepsilon}\right|=O(\varepsilon)$ and $d_{0 \varepsilon} \leq \lambda_{1}$ by (3.19) (if $\varepsilon \leq \varepsilon_{0}$, otherwise the result is clear). This completes the proof of (3.26), and the result of the lemma follows.

The next proposition establishes a lower-bound for the energy on $G_{c_{0} \varepsilon^{\alpha}}$ which is the basis for the proof of Proposition 3.1.

Proposition 3.2. There exist positive constants $K=K(I)$ and $C_{2}=C_{2}(I)$ such that for every $\varepsilon \in(0,1), \alpha \in I$ and $c_{0} \in J_{\alpha, \varepsilon}$ (see Lemma 3.2) there holds

$$
\int_{G_{c_{0} \varepsilon^{\alpha}}}\left|\nabla u_{\varepsilon}\right|^{2}+\frac{W\left(u_{\varepsilon}\right)}{\varepsilon^{2}} \geq \frac{2}{\varepsilon} \int_{\partial G} \Psi(g(\sigma)) d \sigma+K \int_{\partial G} \frac{\left|u_{\varepsilon}\left(\sigma, c_{0} \varepsilon^{\alpha}\right)-\tilde{s}(g(\sigma))\right|^{2}}{\varepsilon^{\alpha}} d \sigma-C_{2} .
$$

Proof. By Lemma 3.5) and (3.23) we have

$$
\int_{G_{c_{0} \varepsilon^{\alpha}}} \frac{\left|\nabla \Psi\left(u_{\varepsilon}\right)\right|^{2}}{W\left(u_{\varepsilon}\right)}+\frac{W\left(u_{\varepsilon}\right)}{\varepsilon^{2}} \geq \frac{2}{\varepsilon} \int_{\partial G} \Psi(g(\sigma)) d \sigma+\int_{G_{c_{0} \varepsilon^{\alpha}}} \frac{\left|\nabla d_{1 \varepsilon}\right|^{2}}{W\left(u_{\varepsilon}\right)+\varepsilon^{2}}-C .
$$

Combining it with (3.12)-(3.13) yields

$$
\begin{aligned}
\int_{G_{c_{0} \varepsilon} \alpha}\left|\nabla u_{\varepsilon}\right|^{2}+\frac{W\left(u_{\varepsilon}\right)}{\varepsilon^{2}} \geq & \frac{2}{\varepsilon} \int_{\partial G} \Psi(g(\sigma)) d \sigma \\
& +\int_{G_{c_{0} \varepsilon^{\alpha}}} \frac{\left|\nabla d_{1 \varepsilon}\right|^{2}}{W\left(u_{\varepsilon}\right)+\varepsilon^{2}}+\beta \int_{G_{c_{0} \varepsilon^{\alpha} \cap G_{0}^{\varepsilon}}}\left|\nabla\left(\tilde{s}\left(u_{\varepsilon}\right)\right)\right|^{2}-C .
\end{aligned}
$$

Fix any $\sigma_{0} \in \partial G$. We distinguish two cases.

Case 1: For all $\delta \in\left(0, c_{0} \varepsilon^{\alpha}\right)$ we have $d_{1 \varepsilon}\left(\sigma_{0}, \delta\right) \leq \lambda_{0}-\lambda_{1}$ (see (1.14)).

In this case, since $d_{0 \varepsilon}\left(\sigma_{0}, \delta\right) \leq \lambda_{1}$ by (3.19), we have $\left(\sigma_{0}, \delta\right) \in G_{0}^{\varepsilon}$ for every $\delta \in$ $\left(0, c_{0} \varepsilon^{\alpha}\right)$ (see (3.11)). Using the Cauchy-Schwarz inequality we get

$$
\beta \int_{0}^{c_{0} \varepsilon^{\alpha}}\left|\nabla\left(\tilde{s}\left(u_{\varepsilon}\left(\sigma_{0}, \delta\right)\right)\right)\right|^{2} d \delta \geq C \frac{\left|\tilde{s}\left(g\left(\sigma_{0}\right)\right)-\tilde{s}\left(u_{\varepsilon}\left(\sigma_{0}, c_{0} \varepsilon^{\alpha}\right)\right)\right|^{2}}{\varepsilon^{\alpha}} .
$$

By (2.6),

$$
\left|u_{\varepsilon}\left(\sigma_{0}, c_{0} \varepsilon^{\alpha}\right)-\tilde{s}\left(u_{\varepsilon}\left(\sigma_{0}, c_{0} \varepsilon^{\alpha}\right)\right)\right|^{2}=O\left(\Psi\left(u_{\varepsilon}\left(\sigma_{0}, c_{0} \varepsilon^{\alpha}\right)\right)\right)=O\left(W\left(u_{\varepsilon}\left(\sigma_{0}, c_{0} \varepsilon^{\alpha}\right)\right)\right) .
$$

So in this case we obtain, for some constants $K_{0}, K_{1}>0$ :

$$
\beta \int_{0}^{c_{0} \varepsilon^{\alpha}}\left|\nabla\left(\tilde{s}\left(u_{\varepsilon}\left(\sigma_{0}, \delta\right)\right)\right)\right|^{2} d \delta \geq K_{0} \frac{\left|u_{\varepsilon}\left(\sigma_{0}, c_{0} \varepsilon^{\alpha}\right)-\tilde{s}\left(g\left(\sigma_{0}\right)\right)\right|^{2}}{\varepsilon^{\alpha}}-K_{1} \frac{W\left(u_{\varepsilon}\left(\sigma_{0}, c_{0} \varepsilon^{\alpha}\right)\right)}{\varepsilon^{\alpha}} .
$$


Case 2: There exists $\delta^{\prime} \in\left(0, c_{0} \varepsilon^{\alpha}\right)$ such that $d_{1 \varepsilon}\left(\sigma_{0}, \delta^{\prime}\right)>\lambda_{0}-\lambda_{1}$.

In this case, since $u_{\varepsilon}$ is bounded thanks to (3.1), we obtain again by the CauchySchwarz inequality that

$$
\begin{aligned}
\int_{0}^{c_{0} \varepsilon^{\alpha}} \frac{\left|\nabla d_{1 \varepsilon}\left(\sigma_{0}, \delta\right)\right|^{2} d \delta}{W\left(u_{\varepsilon}\left(\sigma_{0}, \delta\right)\right)+\varepsilon^{2}} & \geq c \int_{0}^{\delta^{\prime}}\left|\nabla d_{1 \varepsilon}\left(\sigma_{0}, \delta\right)\right|^{2} d \delta \geq c \frac{d_{1, \varepsilon}^{2}\left(\sigma_{0}, \delta^{\prime}\right)}{\delta^{\prime}} \\
& \geq c \frac{\left(\lambda_{0}-\lambda_{1}\right)^{2}}{c_{0} \varepsilon^{\alpha}} \geq K_{2} \frac{\left|u_{\varepsilon}\left(\sigma_{0}, c_{0} \varepsilon^{\alpha}\right)-\tilde{s}\left(g\left(\sigma_{0}\right)\right)\right|^{2}}{\varepsilon^{\alpha}}
\end{aligned}
$$

for some $K_{2}>0$.

Integration over $\sigma_{0} \in \partial G$ of either (3.32) or (3.33) yields for some positive constants $K$ and $\tilde{K}_{1}$ :

$$
\begin{aligned}
& \int_{G_{c_{0} \varepsilon^{\alpha}}} \frac{\left|\nabla d_{1 \varepsilon}\right|^{2}}{W\left(u_{\varepsilon}\right)+\varepsilon^{2}}+\beta \int_{G_{c_{0} \varepsilon^{\alpha}} \cap G_{0}^{\varepsilon}}\left|\nabla\left(\tilde{s}\left(u_{\varepsilon}\right)\right)\right|^{2} \\
& \geq K \int_{\partial G} \frac{\left|u_{\varepsilon}\left(\sigma, c_{0} \varepsilon^{\alpha}\right)-\tilde{s}(g(\sigma))\right|^{2}}{\varepsilon^{\alpha}} d \sigma-\tilde{K}_{1} \int_{\partial G} \frac{W\left(u_{\varepsilon}\left(\sigma, c_{0} \varepsilon^{\alpha}\right)\right)}{\varepsilon^{\alpha}} d \sigma:=I_{1}-I_{2} .
\end{aligned}
$$

Since $I_{2}$ is bounded thanks to (3.6), the result of the lemma follows from (3.31) and (3.34).

Proof of Proposition 3.1. By (1.1)-(1.3) we get that there exists a constant $\kappa>0$ such that

$$
W(z) \geq \kappa \tilde{\delta}^{2}(z), \quad \forall z \in \mathbb{R}^{2} .
$$

Therefore, from Proposition 3.2 we obtain for any $\alpha \in I \subset \subset(1 / 2,1)$ that

$$
\begin{aligned}
& E_{\varepsilon}\left(u_{\varepsilon}\right)-\frac{2}{\varepsilon} \int_{\partial G} \Psi(g(\sigma)) d \sigma \\
& \quad \geq \int_{G \backslash G_{c_{0} \varepsilon^{\alpha}}}\left|\nabla u_{\varepsilon}\right|^{2}+\kappa \frac{\tilde{\delta}^{2}\left(u_{\varepsilon}\right)}{\varepsilon^{2}}+K \int_{\partial G} \frac{\left|u_{\varepsilon}\left(\sigma, c_{0} \varepsilon^{\alpha}\right)-\tilde{s}(g(\sigma))\right|^{2}}{\varepsilon^{\alpha}} d \sigma-C_{2},
\end{aligned}
$$

with $c_{0}, C_{2}$ and $K$ given by Proposition 3.2, By the same proof as that of 2, Proposition 5.1], which deals with the case of $W(u)=F(\tilde{\delta}(u))\left(\right.$ here $\left.F(t)=\kappa t^{2}\right)$, we conclude that

$\int_{G \backslash G_{c_{0} \varepsilon^{\alpha}}}\left|\nabla u_{\varepsilon}\right|^{2}+\kappa \frac{\tilde{\delta}^{2}\left(u_{\varepsilon}\right)}{\varepsilon^{2}}+K \int_{\partial G} \frac{\left|u_{\varepsilon}\left(\sigma, c_{0} \varepsilon^{\alpha}\right)-\tilde{s}(g(\sigma))\right|^{2}}{\varepsilon^{\alpha}} d \sigma \geq D \frac{l^{2}(\Gamma)}{2 \pi} \log \frac{1}{\varepsilon}-C$.

The conclusion of Proposition 3.1 follows from (3.36) and (3.37).

Proof of Theorem 1. It suffices to combine the upper-bound of Proposition 2.1 with the lower-bound of Proposition 3.1.

\section{Convergence of $u_{\varepsilon}$}

The proof of the convergence result Theorem 2 is very similar to that of [2, Theorem 2]. Therefore we shall go over the main steps of the proof, underlying only the modifications of the arguments of [2] which are needed. The next lemma is analogous to [2, Lemma 6.1]. We shall use the notations of Lemma 3.2 . 
Lemma 4.1. Let I be a compact subinterval of $\left(\frac{1}{2}, 1\right)$. Then, there exists a constant $C_{0}>0$ such that for every $\varepsilon \in(0,1), \alpha \in I$ and $c_{0} \in J_{\alpha, \varepsilon}$ we have

$$
\int_{\partial G} \frac{\left|u_{\varepsilon}\left(\sigma, c_{0} \varepsilon^{\alpha}\right)-\tilde{s}(g(\sigma))\right|^{2}}{\varepsilon^{\alpha}} d \sigma \leq C_{0} .
$$

Furthermore, setting

$$
c_{1}=c_{1}(\alpha, \varepsilon)=\inf \left\{c_{0} \in J_{\alpha, \varepsilon}\right\} \quad \text { and } c_{2}=c_{2}(\alpha, \varepsilon)=\sup \left\{c_{0} \in J_{\alpha, \varepsilon}\right\},
$$

we have

$$
\int_{G_{c_{2} \varepsilon^{\alpha}} \backslash G_{c_{1} \varepsilon^{\alpha}}}\left|\nabla u_{\varepsilon}\right|^{2}+\int_{G \backslash G_{c_{1} \varepsilon^{\alpha}}} W\left(u_{\varepsilon}\right) \leq C_{1} .
$$

Proof. By the upper-bound (2.12) and (3.36) we get that

$$
\begin{array}{r}
\int_{G \backslash G_{c_{0} \varepsilon^{\alpha}}}\left|\nabla u_{\varepsilon}\right|^{2}+\kappa \frac{\tilde{\delta}^{2}\left(u_{\varepsilon}\right)}{\varepsilon^{2}}+K \int_{\partial G} \frac{\left|u_{\varepsilon}\left(\sigma, c_{0} \varepsilon^{\alpha}\right)-\tilde{s}(g(\sigma))\right|^{2}}{\varepsilon^{\alpha}} d \sigma \leq D \frac{l^{2}(\Gamma)}{2 \pi} \log \frac{1}{\varepsilon}+C, \\
\forall c_{0} \in J_{\alpha, \varepsilon}, \forall \varepsilon \in(0,1) .
\end{array}
$$

The proof of (3.37) gives the same estimate with $K$ being replaced by $K / 2$, namely (4.5)

$\int_{G \backslash G_{c_{0} \varepsilon^{\alpha}}}\left|\nabla u_{\varepsilon}\right|^{2}+\kappa \frac{\tilde{\delta}^{2}\left(u_{\varepsilon}\right)}{\varepsilon^{2}}+\frac{K}{2} \int_{\partial G} \frac{\left|u_{\varepsilon}\left(\sigma, c_{0} \varepsilon^{\alpha}\right)-\tilde{s}(g(\sigma))\right|^{2}}{\varepsilon^{\alpha}} d \sigma \geq D \frac{l^{2}(\Gamma)}{2 \pi} \log \frac{1}{\varepsilon}-C$.

Combining (4.4) with (4.5) we are led to 4.1).

Similarly, since (3.37) remains valid when $\kappa$ is replaced by $\kappa / 2$, we conclude that

$$
\int_{G \backslash G_{c_{1} \varepsilon^{\alpha}}} \frac{W\left(u_{\varepsilon}\right)}{\varepsilon^{2}} \leq c \int_{G \backslash G_{c_{1} \varepsilon^{\alpha}}} \frac{\tilde{\delta}^{2}\left(u_{\varepsilon}\right)}{\varepsilon^{2}} \leq C,
$$

with $c_{1}$ given by (4.2) (we used (4.1), (3.35) and (3.1)). Finally, to prove the estimate for the first term on the l.h.s. of (4.3), it suffices to notice that the above arguments imply that

$$
D \frac{l^{2}(\Gamma)}{2 \pi} \log \frac{1}{\varepsilon}-C \leq \int_{G \backslash G_{c_{2} \varepsilon^{\alpha}}}\left|\nabla u_{\varepsilon}\right|^{2} \leq \int_{G \backslash G_{c_{1} \varepsilon^{\alpha}}}\left|\nabla u_{\varepsilon}\right|^{2} \leq D \frac{l^{2}(\Gamma)}{2 \pi} \log \frac{1}{\varepsilon}+C .
$$

Fix any interval $I \subset \subset(1 / 2,1)$ and then choose any $\alpha \in I$. Since for every $\varepsilon \in(0,1)$ we have $c_{2}-c_{1} \geq \mu$ (see (4.2) and Lemma 3.2), it follows from (4.3) and Fubini's theorem that there exists $t_{\varepsilon} \in\left(c_{1} \varepsilon^{\alpha}, c_{2} \varepsilon^{\alpha}\right)$ such that

$$
\frac{t_{\varepsilon}}{\varepsilon^{\alpha}} \in J_{\alpha, \varepsilon} \text { and } \int_{\Sigma_{t_{\varepsilon}}}\left|\nabla u_{\varepsilon}\right|^{2} \leq \frac{C}{\varepsilon^{\alpha}} .
$$

Following [2, we shall next define a set of "bad points" $\widetilde{S}_{\varepsilon}$ (a notion that was introduced originally in [5]). We fix a small positive $m$ satisfying

$$
\left\{z \in \mathbb{R}^{2},|\tilde{\delta}(z)|<m\right\} \subset \Omega_{\lambda_{0}}(\text { see (1.11) }),
$$


and set

$$
\begin{array}{r}
\widetilde{S}_{\varepsilon}=\left\{x \in G \backslash G_{t_{\varepsilon}}:\left|\tilde{\delta}\left(u_{\varepsilon}(x)\right)\right|>m\right\} \cup\left\{x \in \Sigma_{t_{\varepsilon}}:\left|u_{\varepsilon}(x)-\tilde{s}(g(\sigma(x)))\right|>m\right\} \\
:=\widetilde{S}_{\varepsilon, 1} \cup \widetilde{S}_{\varepsilon, 2} .
\end{array}
$$

Our next objective is to cover the set $\widetilde{S}_{\varepsilon, 1}$ by a finite collection of discs/ "half discs" with radii of the order $\varepsilon$, and to cover the set $\widetilde{S}_{\varepsilon, 2}$ by a finite collection of "half discs" with radii of the order $\varepsilon^{\alpha}$. A "half disc" is by definition a set of the form $B^{+}(\sigma, r)=B(\sigma, r) \cap\left(G \backslash G_{t_{\varepsilon}}\right)$, for some $\sigma \in \Sigma_{t_{\varepsilon}}$ and $r>0$. We shall denote it for $x \in G \backslash G_{t_{\varepsilon}}: \delta_{1}(x)=\operatorname{dist}\left(x, \Sigma_{t_{\varepsilon}}\right)$. As in [2, Lemma 6.2] we have

Lemma 4.2. There exist a constant $k_{1}>0$ and an integer $N_{1}$, both independent of $\varepsilon$, such that, the collection of mutually disjoint discs/half discs $\left\{B\left(x_{i}^{\varepsilon}, \lambda_{\varepsilon} \varepsilon\right) \cap(G \backslash\right.$ $\left.\left.G_{t_{\varepsilon}}\right)\right\}_{i=1}^{N_{1}(\varepsilon)}$ satisfies

$$
\widetilde{S}_{\varepsilon, 1} \subset \bigcup_{i=1}^{N_{1}(\varepsilon)} B\left(x_{i}^{\varepsilon}, \lambda_{\varepsilon} \varepsilon\right) \cap\left(G \backslash G_{t_{\varepsilon}}\right),
$$

with $\lambda_{\varepsilon} \leq k_{1}$ and $N_{1}(\varepsilon) \leq N_{1}$, such that for each $i$ either $\delta_{1}\left(x_{i}^{\varepsilon}\right) \geq 2 \lambda_{\varepsilon} \varepsilon$ or $x_{i}^{\varepsilon} \in$ $\partial \Sigma_{t_{\varepsilon}}$. Moreover, there exist $k_{2}$ and $N_{2}$ (independent of $\varepsilon$ ), and for each $\varepsilon>0$, a collection of mutually disjoint half bad discs $\left\{B^{+}\left(y_{j}^{\varepsilon}, \gamma_{\varepsilon} t_{\varepsilon}\right)\right\}_{j=1}^{N_{2}(\varepsilon)}$, with $y_{j}^{\varepsilon} \in \Sigma_{t_{\varepsilon}}$, that satisfies

$$
\widetilde{S}_{\varepsilon, 2} \subset \bigcup_{j=1}^{N_{2}(\varepsilon)} B^{+}\left(y_{j}^{\varepsilon}, \gamma_{\varepsilon} t_{\varepsilon}\right),
$$

with $\gamma_{\varepsilon} \leq k_{2}$ and $N_{2}(\varepsilon) \leq N_{2}$. Further, we can construct such collections with the property that for each $1 \leq i \leq N_{1}(\varepsilon)$ and $1 \leq j \leq N_{2}(\varepsilon)$,

$$
\text { either } B\left(x_{i}^{\varepsilon}, \lambda_{\varepsilon} \varepsilon\right) \cap B^{+}\left(y_{j}^{\varepsilon}, \gamma_{\varepsilon} t_{\varepsilon}\right)=\emptyset \quad \text { or } \quad B\left(x_{i}^{\varepsilon}, \lambda_{\varepsilon} \varepsilon\right) \cap\left(G \backslash G_{t_{\varepsilon}}\right) \subset B^{+}\left(y_{j}^{\varepsilon}, \gamma_{\varepsilon} t_{\varepsilon}\right) \text {. }
$$

The proof is identical to the one given in 2, so we omit it. We just mention that in order to prove that $\widetilde{S}_{\varepsilon, 2}$ can be covered by a bounded number (i.e., uniformly in $\varepsilon)$ of half bad discs $\left\{B^{+}\left(y_{j}^{\varepsilon}, \gamma_{\varepsilon} t_{\varepsilon}\right)\right\}_{j=1}^{N_{2}(\varepsilon)}$ we need to know that there exists $\mu_{2}>0$ such that

$$
x \in \widetilde{S}_{\varepsilon, 2} \Longrightarrow\left|u_{\varepsilon}(y)-\tilde{g}(y)\right|>m / 2, \quad \forall y \in \Sigma_{t_{\varepsilon}} \text { with }|y-x| \leq \mu_{2} t_{\varepsilon} .
$$

The proof of (4.11) follows from the estimate

$$
\left|u_{\varepsilon}(x)-u_{\varepsilon}(y)\right|^{2} \leq C\left(\int_{\Sigma_{t_{\varepsilon}}}\left|\nabla u_{\varepsilon}\right|^{2}\right)|x-y| \leq\left(\frac{C}{t_{\varepsilon}}\right)|x-y|, \quad \forall x, y \in \Sigma_{t_{\varepsilon}},
$$

which is a consequence of the Cauchy-Schwarz inequality and the choice of $t_{\varepsilon}$ in (4.6).

Next, as in 2 we show that the degree of $u_{\varepsilon}$ on the boundary of each of the discs/half discs is bounded, uniformly in $\varepsilon$ (see [2] for definition of the degree on the boundary of a half-disc ). Then, we can identify the limits of the centers of the discs/half discs (for a subsequence $\varepsilon_{n} \rightarrow 0$ ) and obtain the following result, whose proof is identical to that of [2, Proposition 6.1] (see in particular (6.30) in [2]). 
Proposition 4.1. For a subsequence $\varepsilon_{n} \rightarrow 0$, there exist $D$ distinct points $a_{1}, \ldots$, $a_{D} \in G$ such that the degree of $u_{\varepsilon_{n}}$ around each $a_{j}$ is 1 and for every $r$ satisfying (4.12) $0<r<\frac{1}{2} \min \left(\min \left\{\left|a_{i}-a_{j}\right|: i \neq j\right\}, \min \left\{\operatorname{dist}\left(a_{j}, \partial G\right): j=1, \ldots, D\right\}\right)$, we have

$$
E_{\varepsilon_{n}}\left(u_{\varepsilon_{n}}, G \backslash\left(G_{r} \cup \bigcup_{j=1}^{D} B\left(a_{j}, r\right)\right)\right) \leq C(r) .
$$

Now we are ready to complete the proof of Theorem 2 .

Proof of Theorem 2. By (4.13) we get (possibly after passing to a further subsequence) that

$$
u_{\varepsilon_{n}} \rightarrow u_{*} \text { weakly in } H_{\mathrm{loc}}^{1}\left(G \backslash\left\{a_{1}, \ldots, a_{D}\right\}\right),
$$

for some $u_{*} \in H_{\text {loc }}^{1}\left(G \backslash\left\{a_{1}, \ldots, a_{D}\right\}, \Gamma\right)$. In order to obtain strong convergence in $H_{\text {loc }}^{1}\left(G \backslash\left\{a_{1}, \ldots, a_{D}\right\}\right)$ we fix any disc $B\left(x_{0}, r\right) \subset \subset G \backslash\left\{a_{1}, \ldots, a_{D}\right\}$. By (4.13) we have $E_{\varepsilon_{n}}\left(u_{\varepsilon_{n}}, B\left(x_{0}, r\right)\right) \leq C$. Using Fubini's theorem we can find $r_{0} \in(r / 2, r)$ such that (by passing to a subsequence if necessary)

$$
\int_{\partial B\left(x_{0}, r_{0}\right)}\left|\nabla u_{\varepsilon_{n}}\right|^{2}+\frac{W\left(u_{\varepsilon_{n}}\right)}{\varepsilon_{n}^{2}} \leq C, \forall n .
$$

Applying the argument of [4] yields that

$$
u_{\varepsilon_{n}} \rightarrow u_{*} \text { in } H^{1}\left(B\left(x_{0}, r_{0}\right)\right) \text { and } \tilde{\delta}\left(u_{\varepsilon_{n}}\right) \rightarrow 0 \text { uniformly on } \overline{B\left(x_{0}, r_{0}\right)} \text {. }
$$

Moreover, $u_{*}$ is a $\Gamma$-valued map which is a local minimizer of the Dirichlet energy among such maps in $G \backslash\left\{a_{1}, \ldots, a_{D}\right\}$. We can write then, locally in $G \backslash\left\{a_{1}, \ldots, a_{D}\right\}$, $u_{*}=\tau\left(e^{i \phi_{*}}\right)$ (see (1.17) $)$ where $\phi_{*}$ is a harmonic function.

Next we shall apply the argument of Steps A.3 and A.4 from the proof of [4, Theorem 1], and more specifically the variant in [2, to show that

$\left\{u_{\varepsilon_{n}}\right\}$ is bounded in $H_{\mathrm{loc}}^{2}\left(B\left(x_{0}, r_{0}\right)\right)$ and $\left\{\nabla u_{\varepsilon_{n}}\right\}$ is bounded in $L_{\mathrm{loc}}^{\infty}\left(B\left(x_{0}, r_{0}\right)\right)$.

We first introduce a new coordinate system in a neighborhood of $\Gamma$. From our assumptions on $\Gamma$ and $W$ it follows that for some small $\eta_{1}>0$, the set $\Gamma_{\eta_{1}}$ (see (2.2) ) is covered by a system of nonintersecting gradient lines of $W$. For each $x \in \Gamma_{\eta_{1}}$ we associate the coordinate $\bar{\sigma}=\bar{\sigma}(x)$ which is the intersection of the gradient line passing through $x$ with $\Gamma$. The second coordinate is by definition $\bar{\delta}=\bar{\delta}(x)$ satisfying:

(i) $|\bar{\delta}(x)|=\sqrt{W(x)}$,

(ii) $\operatorname{sgn} \bar{\delta}(x)$ is negative inside $\Gamma$ and positive outside.

Since $\Gamma$ and $W$ are both of class $C^{4}$, it follows that $\bar{\sigma}$ and $\bar{\delta}$ are of class $C^{2}$ in $\Gamma_{\eta_{1}}$. Using $|\nabla \bar{\delta}|=\frac{1}{2} W^{-1 / 2}|\nabla W|$, together with (2.4) and (5.1) we obtain that

$$
\alpha_{1} \leq|\nabla \bar{\delta}(x)| \leq \alpha_{2}, \forall x \in \Gamma_{\eta_{1}},
$$

for some positive constants $\alpha_{1}, \alpha_{2}$. Note also that $W_{\bar{\delta} \bar{\delta}}=2$ in a neighborhood of $\Gamma$.

Dropping for simplicity the subscript $\varepsilon_{n}$, we set $A=\frac{1}{2}|\nabla u|^{2}$, so that

$$
\Delta A=\left|D^{2} u\right|^{2}+\sum_{i=1}^{2} u_{x_{i}} \Delta\left(u_{x_{i}}\right) .
$$


Writing $u=\left(u_{1}, u_{2}\right)$ we get by a direct computation on $B\left(x_{0}, r_{0}\right)$, for $i=1,2$ and $\varepsilon_{n}$ small enough, that

$$
\begin{aligned}
u_{x_{i}} \Delta\left(u_{x_{i}}\right)= & \frac{W_{\bar{\delta}}(u)}{2 \varepsilon_{n}^{2}}\left(\bar{\delta}_{u_{1} u_{1}}\left(u_{1}\right)_{x_{i}}^{2}+2 \bar{\delta}_{u_{1} u_{2}}\left(u_{1}\right)_{x_{i}}\left(u_{2}\right)_{x_{i}}+\bar{\delta}_{u_{2} u_{2}}\left(u_{2}\right)_{x_{i}}^{2}\right) \\
& +\frac{W_{\bar{\delta} \bar{\delta}}}{2 \varepsilon_{n}^{2}}\left(\bar{\delta}_{u_{1}}\left(u_{1}\right)_{x_{i}}+\bar{\delta}_{u_{2}}\left(u_{2}\right)_{x_{i}}\right)^{2} \\
& \geq \frac{W_{\bar{\delta}}(u)}{2 \varepsilon_{n}^{2}}\left(\bar{\delta}_{u_{1} u_{1}}\left(u_{1}\right)_{x_{i}}^{2}+2 \bar{\delta}_{u_{1} u_{2}}\left(u_{1}\right)_{x_{i}}\left(u_{2}\right)_{x_{i}}+\bar{\delta}_{u_{2} u_{2}}\left(u_{2}\right)_{x_{i}}^{2}\right) .
\end{aligned}
$$

Noting that by (3.3)

$$
|\Delta u|=\frac{|\nabla W(u)|}{2 \varepsilon_{n}^{2}}=\frac{\left|W_{\bar{\delta}}(u)\right||\nabla \bar{\delta}|}{2 \varepsilon_{n}^{2}},
$$

we infer from (4.18), (4.19) and (4.17) that for some $c>0$ we have

$$
\Delta A \geq\left|D^{2} u\right|^{2}-c|\Delta u||\nabla u|^{2} .
$$

Since $|\Delta u| \leq \sqrt{2}\left|D^{2} u\right|$ we get by (4.20) and the Cauchy-Schwarz inequality that

$$
-\Delta A+\left|D^{2} u\right|^{2} \leq \frac{1}{2}\left|D^{2} u\right|^{2}+4 c A^{2} .
$$

From the Bochner-type inequality (4.21) we deduce (4.16) as in [4. In particular we obtain that

$$
u_{\varepsilon_{n}} \rightarrow u_{*} \text { in } C_{\mathrm{loc}}^{\alpha}\left(G \backslash\left\{a_{1}, \ldots, a_{D}\right\}\right) .
$$

Next we show that the trace of $u_{*}$ on $\partial G$ equals $\tilde{s}(g)$. Note first that thanks to (4.13), $u_{*} \in H^{1}\left(G_{r}\right)$ for $r$ satisfying (4.12), so it has a trace in $H^{1 / 2}\left(\partial G, S^{1}\right)$. Fix a point $y \in \partial G$ which is not a limit point of the centers of discs/half discs given by Lemma 4.2. It is enough to show that the trace of $u_{*}$ equals $\tilde{s}(g)$ in a small boundary interval around each such $y$. Next fix some $r>0$ small enough so that $B(y, r) \cap G$ does not intersect any of the discs/half discs. For each $n$ we shall now define a map $w_{\varepsilon_{n}}$ on the domain $B(y, r) \cap G$ as follows. On $B(y, r) \cap\left(G \backslash G_{t_{\varepsilon_{n}}}\right)$ we simply set $w_{\varepsilon_{n}}=u_{\varepsilon_{n}}\left(t_{\varepsilon_{n}}\right.$ is given in (4.6) $)$. On the remaining part, namely $B(y, r) \cap G_{t_{\varepsilon_{n}}}$, we set, using the $(\sigma, \delta)$-coordinates (see the beginning of Section 2):

$$
w_{\varepsilon_{n}}(\sigma, \delta)=\left(\delta / t_{\varepsilon_{n}}\right) u_{\varepsilon_{n}}\left(\sigma, t_{\varepsilon_{n}}\right)+\left(1-\delta / t_{\varepsilon_{n}}\right) \tilde{s}(g(\sigma)) .
$$

A simple computation, using (4.1) and (4.6), gives that

$$
\int_{B(y, r) \cap G_{t_{\varepsilon_{n}}}}\left|\nabla w_{\varepsilon_{n}}\right|^{2}+\left|w_{\varepsilon_{n}}\right|^{2} \leq C, \text { uniformly in } n .
$$

By (4.14) we conclude then that $w_{\varepsilon_{n}} \rightarrow u_{*}$ in $H^{1}(B(y, r) \cap G)$. Since the trace of each $w_{\varepsilon_{n}}$ on $B(y, r) \cap \partial G$ is $\tilde{s}(g)$, we conclude that the same is true for $u_{*}$.

In order to complete the proof of Theorem 2 we need to show that $u_{*}$ is of the form given in (1.18). First, applying an argument of Struwe 12 we obtain that $u_{*} \in W^{1, p}\left(G, S^{1}\right)$ for every $p \in[1,2)$. Then, it follows from [5], Remark I.1] that $v_{*}:=\tau^{-1}\left(u_{*}\right)$ must be of the form

$$
v_{*}(z)=e^{i \phi_{0}} \prod_{j=1}^{D}\left(\frac{z-a_{j}}{\left|z-a_{j}\right|}\right) e^{i\left(\sum_{j=1}^{D} c_{j} \log \left|z-a_{j}\right|\right)},
$$


for some constants $c_{1}, \ldots, c_{D}$, where $\phi_{0}$ is a smooth harmonic function. Fix any $r$ satisfying (4.12). Then by (4.23) we have

$$
\int_{B\left(a_{j}, r\right) \backslash B\left(a_{j}, \eta\right)}\left|\nabla u_{*}\right|^{2}=\left(1+c_{j}^{2}\right) \frac{l^{2}(\Gamma)}{2 \pi} \log \frac{1}{\eta}+O(1), \forall \eta<r, j=1, \ldots, D .
$$

On the other hand, the argument leading to Proposition 4.1 gives

$$
\int_{B\left(a_{j}, r\right) \backslash B\left(a_{j}, \eta\right)}\left|\nabla u_{*}\right|^{2} \leq \frac{l^{2}(\Gamma)}{2 \pi} \log \frac{1}{\eta}+O(1), \forall \eta<r, j=1, \ldots, D .
$$

Combining (4.24) with (4.25) we get that $c_{j}=0, \forall j$, and it follows that $u_{*}$ has the desired form.

Remark 4.1. Repeating the argument from [2, it is not difficult to prove that the limiting configuration $\vec{a}=\left(a_{1}, \ldots, a_{D}\right)$ in Theorem 2 is a minimizing configuration for a certain renormalized energy, as defined in [5] (see [2 for details).

\section{Appendix: Local Regularity of $\Psi$}

The main objective of this Appendix is to derive a regularity result for $\Psi$ in a neighborhood of $\Gamma$. Differentiating (2.4) gives the following formulas for the derivatives of $W$ w.r.t. the variables $\tilde{\sigma}$ and $\tilde{\delta}$ (that were defined at the beginning of Section 2) in $\Gamma_{\eta_{0}}$ :

$$
\frac{\partial W}{\partial \tilde{\sigma}}=a_{\tilde{\sigma}}(\tilde{\sigma}, \tilde{\delta}) \tilde{\delta}^{2} \quad \text { and } \quad \frac{\partial W}{\partial \tilde{\delta}}=a_{\tilde{\delta}}(\tilde{\sigma}, \tilde{\delta}) \tilde{\delta}^{2}+2 a(\tilde{\sigma}, \tilde{\delta}) \tilde{\delta} .
$$

We may write

$$
\nabla W=\frac{\partial W}{\partial \tilde{\sigma}} \vec{\tau}+\frac{\partial W}{\partial \tilde{\delta}} \vec{n},
$$

with $\vec{n}=\nabla \tilde{\delta}$ and $\vec{\tau}=\nabla \tilde{\sigma}$. By our regularity assumptions on $\Gamma$ there exist continuous functions $c_{1}(x), c_{2}(x), c_{3}(x), c_{4}(x)$ such that

$$
\frac{\partial \vec{n}}{\partial \tilde{\sigma}}=c_{1}(x) \vec{\tau}, \quad \frac{\partial \vec{\tau}}{\partial \tilde{\sigma}}=c_{2}(x) \vec{n}+c_{3}(x) \vec{\tau} \quad \text { and } \quad \frac{\partial \vec{\tau}}{\partial \tilde{\delta}}=c_{4}(x) \vec{\tau} .
$$

Note that for $x \in \Gamma$ we have $c_{1}(x)=c(x)=$ the curvature of $\Gamma$ at $x$. From (5.1)(5.2) we derive that

$$
\begin{aligned}
\frac{\partial}{\partial \tilde{\sigma}} \nabla W= & \left(a_{\tilde{\sigma} \tilde{\sigma}} \tilde{\delta}^{2}+c_{1}(x)\left(a_{\tilde{\delta}} \tilde{\delta}^{2}+2 a \tilde{\delta}\right)+c_{3}(x) a_{\tilde{\sigma}} \tilde{\delta}^{2}\right) \vec{\tau} \\
& +\left(\left(a_{\tilde{\sigma} \tilde{\delta}}+c_{2}(x) a_{\tilde{\sigma}}\right) \tilde{\delta}^{2}+2 a_{\tilde{\sigma}} \tilde{\delta}\right) \vec{n}=O(\tilde{\delta})
\end{aligned}
$$

and

(5.4) $\frac{\partial}{\partial \tilde{\delta}} \nabla W=\left(a_{\tilde{\sigma} \tilde{\delta}} \tilde{\delta}^{2}+2 a_{\tilde{\sigma}} \tilde{\delta}+c_{4}(x) a_{\tilde{\sigma}} \tilde{\delta}^{2}\right) \vec{\tau}+\left(a_{\tilde{\delta} \tilde{\delta}} \tilde{\delta}^{2}+4 a_{\tilde{\delta}} \tilde{\delta}+2 a\right) \vec{n}=2 a \vec{n}+O(\tilde{\delta})$.

The main result of this Appendix is the following

Proposition 5.1. There exists $\lambda_{0}>0$ such that the equation

$$
\left\{\begin{array}{l}
|\nabla U|^{2}=W, \\
U=0 \text { on } \Gamma,
\end{array}\right.
$$

has a unique positive $C^{2}$-solution $U$ in $\bar{\Omega}_{\lambda_{0}}$. Moreover, this solution coincides in $\bar{\Omega}_{\lambda_{0}}$ with $\Psi$, and $\bar{\Omega}_{\lambda_{0}}$ can be covered by a system of gradient lines of $\Psi$, given by the images of $\left\{\gamma^{ \pm} x_{0}\right\}_{x_{0} \in \Gamma}$ (see (2.10) $-(2.11)$ ). 
In the nondegenerate case, the proof of such a result is classical via the characteristic method; see [6, Section 3.2]. We shall use a variant of this method in order to overcome the difficulty caused by the degeneracy of the problem. Define a Hamiltonian

$$
H(X, P)=|P|^{2}-W(X),
$$

so that the first equation in (5.5) can be written as $H(x, \nabla U)=0$. We are looking for a solution $(X, P): \Gamma \times(-\infty, c) \rightarrow \Gamma_{\eta} \times \mathbb{R}^{2}$, for some small $\eta>0$ (see (2.2) ) of the characteristics system

$$
\left\{\begin{array}{l}
X\left(x_{0},-\infty\right)=x_{0}, \forall x_{0} \in \Gamma, \\
\dot{X}=\frac{\partial H}{\partial P}=2 P \\
\dot{P}=-\frac{\partial H}{\partial X}=\nabla W(X)
\end{array}\right.
$$

where the dot represents a derivative w.r.t. the variable $t$. The construction of a solution $U$ from $(X, P)$ is then standard; see (5.36) below. In order to define a problem on a bounded domain we make the change of variables $r=e^{\alpha t}$, where $\alpha=\alpha\left(x_{0}\right)=2 \sqrt{a\left(x_{0}\right)}$ (see (2.4)). Using this new variable, (5.7) becomes

$$
\left\{\begin{array}{l}
X\left(x_{0}, 0\right)=x_{0} \text { and } P\left(x_{0}, 0\right)=0, \forall x_{0} \in \Gamma, \\
\frac{\partial X}{\partial r}=\frac{2 P}{\alpha\left(x_{0}\right) r} \\
\frac{\partial P}{\partial r}=\frac{\nabla W(X)}{\alpha\left(x_{0}\right) r} .
\end{array}\right.
$$

We shall construct a solution $X$ of $(5.8)$ with image in a one-sided neighborhood of $\Gamma$, of the form $\Gamma_{\eta}^{+}=\left\{x \in \mathbb{R}^{2}: \tilde{\delta}(x) \in[0, \eta)\right\}$, but an analogous argument will also give a solution on the other side of $\Gamma$, namely in $\Gamma_{\eta}^{-}=\left\{x \in \mathbb{R}^{2}: \tilde{\delta}(x) \in(-\eta, 0]\right\}$.

Integrating the equations in (5.8) yields an equivalent form:

$$
X\left(x_{0}, r\right)-x_{0}=\int_{0}^{r} \frac{2 P\left(x_{0}, s\right)}{\alpha\left(x_{0}\right) s} d s, \quad P\left(x_{0}, r\right)=\int_{0}^{r} \frac{\nabla W\left(X\left(x_{0}, s\right)\right)}{\alpha\left(x_{0}\right) s} d s .
$$

Let $Y$ and $Q$ be defined by

$$
Y\left(x_{0}, r\right)=\frac{X\left(x_{0}, r\right)-x_{0}}{r} \text { and } Q\left(x_{0}, r\right)=\frac{P\left(x_{0}, r\right)}{r} .
$$

If $Q$ and $Y$ are associated with a solution $(X, P)$ to (5.9), then by (5.3)-(5.4) it follows that

$$
\begin{aligned}
Q\left(x_{0}, r\right) & =\frac{1}{r} \int_{0}^{r} \frac{\nabla W\left(x_{0}+s Y\left(x_{0}, s\right)\right)}{\alpha\left(x_{0}\right) s} d s \\
& =\frac{1}{\alpha\left(x_{0}\right) r} \int_{0}^{r} \frac{1}{s}\left(D^{2} W\left(x_{0}\right)\left(s Y\left(x_{0}, s\right)+s o\left(\left|Y\left(x_{0}, s\right)\right|\right)\right)\right) d s \\
& =\frac{\vec{n}}{\alpha\left(x_{0}\right) r} \int_{0}^{r} 2 a\left(x_{0}\right) Y\left(x_{0}, s\right) \cdot \vec{n} d s+o(1)
\end{aligned}
$$

and

$$
Y\left(x_{0}, r\right)=\frac{1}{\alpha\left(x_{0}\right) r} \int_{0}^{r} 2 Q\left(x_{0}, s\right) d s .
$$

From (5.11) - (5.12) we deduce that

$$
\left\{\begin{array}{l}
Q\left(x_{0}, 0\right)=\sqrt{a\left(x_{0}\right)}\left(Y\left(x_{0}, 0\right) \cdot \vec{n}\right) \vec{n} \\
Y\left(x_{0}, 0\right)=\frac{Q\left(x_{0}, 0\right)}{\sqrt{a\left(x_{0}\right)}}
\end{array}\right.
$$


Thus (5.13) implies a compatibility condition on the initial values $Q\left(x_{0}, 0\right), Y\left(x_{0}, 0\right)$. We deduce in particular that $Y\left(x_{0}, 0\right)$ must be parallel to $\vec{n}\left(x_{0}\right)$, and we may choose $Y\left(x_{0}, 0\right)=\vec{n}\left(x_{0}\right)$ (and then necessarily $Q\left(x_{0}, 0\right)=\sqrt{a\left(x_{0}\right)} \vec{n}\left(x_{0}\right)$ ).

Next we introduce yet another pair of unknowns, $\widetilde{Y}, \widetilde{Q}$, by

$$
\left\{\begin{array}{l}
Y\left(x_{0}, r\right)=\vec{n}\left(x_{0}\right)+r \widetilde{Y}\left(x_{0}, r\right), \\
Q\left(x_{0}, r\right)=\sqrt{a\left(x_{0}\right)}\left(\vec{n}\left(x_{0}\right)+r \widetilde{Q}\left(x_{0}, r\right)\right) .
\end{array}\right.
$$

From (5.11) - (5.12) we obtain the following system of equations that must be satisfied by $\widetilde{Y}$ and $\widetilde{Q}$ :

$$
\widetilde{Y}\left(x_{0}, r\right)=\frac{1}{r^{2}} \int_{0}^{r}\left(\frac{1}{\sqrt{a\left(x_{0}\right)}} Q\left(x_{0}, s\right)-\vec{n}\left(x_{0}\right)\right) d s=\frac{1}{r^{2}} \int_{0}^{r} s \widetilde{Q}\left(x_{0}, s\right) d s,
$$

and

$$
\begin{aligned}
\widetilde{Q}\left(x_{0}, r\right) & =\frac{1}{r \sqrt{a\left(x_{0}\right)}}\left(Q\left(x_{0}, r\right)-\sqrt{a\left(x_{0}\right)} \vec{n}\left(x_{0}\right)\right) \\
& =\frac{1}{2 a\left(x_{0}\right) r^{2}} \int_{0}^{r}\left(\frac{1}{s} \nabla W\left(x_{0}+s \vec{n}\left(x_{0}\right)+s^{2} \widetilde{Y}\left(x_{0}, s\right)\right)-2 a\left(x_{0}\right) \vec{n}\left(x_{0}\right)\right) d s .
\end{aligned}
$$

Denoting by $T_{1}(\widetilde{Y}, \widetilde{Q})$ the r.h.s. of $\left(\underline{5.15)}\right.$ and by $T_{2}(\widetilde{Y}, \widetilde{Q})$ the r.h.s. of (5.16), we define a map $T=\left(T_{1}, T_{2}\right)$ from $\left(C\left(\Gamma \times[0, R], \mathbb{R}^{2}\right)\right)^{2}$ to itself by $T(\widetilde{Y}, \widetilde{Q})=$ $\left(T_{1}(\widetilde{Y}, \widetilde{Q}), T_{2}(\widetilde{Y}, \widetilde{Q})\right)$. Clearly $(\widetilde{Y}, \widetilde{Q})$ is a solution to (5.15) (5.16) if and only it is a fixed point of $T$. Next we claim

Lemma 5.1. For $R>0$ small enough, $T$ is a strict contraction w.r.t. the $\|\cdot\|_{\infty}$ norm (and therefore has a unique fixed point).

Proof. Consider any $(\tilde{Y}, \widetilde{Q}),\left(\widetilde{Y}^{\prime}, \widetilde{Q}^{\prime}\right) \in\left(C\left(\Gamma \times[0, R], \mathbb{R}^{2}\right)\right)^{2}$. Using (5.15) we get, for $R$ small enough,

$$
\begin{aligned}
\left\|T_{1}(\widetilde{Y}, \widetilde{Q})-T_{1}\left(\widetilde{Y}^{\prime}, \widetilde{Q}^{\prime}\right)\right\|_{\infty} & =\sup _{\substack{0<r<R \\
x_{0} \in \Gamma}} \frac{1}{r^{2}}\left|\int_{0}^{r} s\left(\widetilde{Q}-\widetilde{Q}^{\prime}\right)\left(x_{0}, s\right) d s\right| \leq \frac{1}{2}\left\|\widetilde{Q}-\widetilde{Q}^{\prime}\right\|_{\infty} \\
& \leq \frac{1}{2}\left\|(\widetilde{Y}, \widetilde{Q})-\left(\widetilde{Y}^{\prime}, \widetilde{Q}^{\prime}\right)\right\|_{\infty} .
\end{aligned}
$$

From (5.16) we obtain that

$$
\begin{aligned}
& \left\|T_{2}(\tilde{Y}, \widetilde{Q})-T_{2}\left(\tilde{Y}^{\prime}, \widetilde{Q}^{\prime}\right)\right\|_{\infty} \\
& =\sup _{\substack{0<r<R \\
x_{0} \in \Gamma}} \frac{1}{2 a\left(x_{0}\right) r^{2}} \mid \int_{0}^{r}\left(\nabla W\left(x_{0}+s \vec{n}\left(x_{0}\right)+s^{2} \widetilde{Y}\left(x_{0}, s\right)\right)\right. \\
& \left.\quad-\nabla W\left(x_{0}+s \vec{n}\left(x_{0}\right)+s^{2} \widetilde{Y}^{\prime}\left(x_{0}, s\right)\right)\right) \frac{d s}{s} \mid .
\end{aligned}
$$

By (5.3) -(5.4) we get that

$\left|\nabla W\left(x_{0}+s \vec{n}\left(x_{0}\right)+s^{2} \tilde{Y}\right)-\nabla W\left(x_{0}+s \vec{n}\left(x_{0}\right)+s^{2} \tilde{Y}^{\prime}\right)\right| \leq\left(2 a\left(x_{0}\right) s^{2}+O\left(s^{3}\right)\right) \cdot\left|\tilde{Y}-\tilde{Y}^{\prime}\right|$. 
Integration on $s$ gives, for every $r \in(0, R)$,

$$
\begin{aligned}
\frac{1}{2 a\left(x_{0}\right) r^{2}} \int_{0}^{r} \frac{1}{s} \mid \nabla W\left(x_{0}+s \vec{n}\left(x_{0}\right)+s^{2} \widetilde{Y}\right)-\nabla W\left(x_{0}\right. & \left.+s \vec{n}\left(x_{0}\right)+s^{2} \widetilde{Y}^{\prime}\right) \mid d s \\
\leq & \left(\frac{1}{2}+O(R)\right)\left\|\widetilde{Y}-\widetilde{Y}^{\prime}\right\|_{\infty},
\end{aligned}
$$

which plugged in (5.18) yields for $R$ small enough

$$
\left\|T_{2}(\widetilde{Y}, \widetilde{Q})-T_{2}\left(\widetilde{Y}^{\prime}, \widetilde{Q}^{\prime}\right)\right\|_{\infty} \leq \frac{3}{4}\left\|(\widetilde{Y}, \widetilde{Q})-\left(\widetilde{Y}^{\prime}, \widetilde{Q}^{\prime}\right)\right\|_{\infty} .
$$

The result of the lemma clearly follows from (5.17) and (5.19).

Fix $R_{0}>0$ for which the conclusion of Lemma 5.1 holds and let $\left(\widetilde{Y}_{\infty}, \widetilde{Q}_{\infty}\right) \in$ $\left(C\left(\Gamma \times\left[0, R_{0}\right], \mathbb{R}^{2}\right)\right)^{2}$ be the fixed point of $T$ given by Lemma 5.1. The next lemma establishes improved regularity for $\widetilde{Y}_{\infty}$ and $\widetilde{Q}_{\infty}$. To study the regularity w.r.t. the first variable we examine the derivative w.r.t. the variable $\tilde{\sigma}_{0}\left(x_{0}=\gamma\left(\tilde{\sigma}_{0}\right)\right.$; see the definition after (2.3) $)$.

Lemma 5.2. There exists $0<R_{1} \leq R_{0}$ such that the functions $\widetilde{Y}_{\infty}$ and $\widetilde{Q}_{\infty}$ have continuous derivatives in the variable $\tilde{\sigma}_{0}$ on $\Gamma \times\left[0, R_{1}\right]$.

Proof. Consider $\widetilde{Y}_{0}, \widetilde{Q}_{0} \in C^{1}\left(\Gamma \times\left[0, R_{0}\right], \mathbb{R}^{2}\right)$ and the sequence of iterates

$$
\left\{\left(\widetilde{Y}_{n}, \widetilde{Q}_{n}\right)\right\}_{n=0}^{\infty}=\left\{T^{n}\left(\widetilde{Y}_{0}, \widetilde{Q}_{0}\right)\right\}_{n=0}^{\infty}
$$

which converges uniformly to the fixed point $\left(\widetilde{Y}_{\infty}, \widetilde{Q}_{\infty}\right)$ of $T$. We will show that the sequence $\left\{\left(\frac{\partial \widetilde{Y}_{n}}{\partial \tilde{\sigma}_{0}}, \frac{\partial \widetilde{Q}_{n}}{\partial \tilde{\sigma}_{0}}\right)\right\}_{n=0}^{\infty}$ is uniformly converging too. We next prove a uniform bound for the $\tilde{\sigma}_{0}$-derivative of the sequence, i.e., that there exists a constant $K>0$ such that, on $\Gamma \times\left[0, R_{0}^{\prime}\right]$, with $0<R_{0}^{\prime} \leq R_{0}$, we have

$$
\left\|\frac{\partial \widetilde{Y}_{n}}{\partial \tilde{\sigma}_{0}}\right\|_{\infty},\left\|\frac{\partial \widetilde{Q}_{n}}{\partial \tilde{\sigma}_{0}}\right\|_{\infty} \leq K, \forall n
$$

Note first that

$$
\frac{\partial \widetilde{Y}_{n+1}}{\partial \tilde{\sigma}_{0}}=\frac{\partial}{\partial \tilde{\sigma}_{0}} T_{1}\left(\widetilde{Y}_{n}, \widetilde{Q}_{n}\right)=\frac{1}{r^{2}} \int_{0}^{r} s \frac{\partial \widetilde{Q}\left(\gamma\left(\tilde{\sigma}_{0}\right), s\right)}{\partial \tilde{\sigma}_{0}} d s
$$

implies that

$$
\left\|\frac{\partial \widetilde{Y}_{n+1}}{\partial \tilde{\sigma}_{0}}\right\|_{\infty} \leq \frac{1}{2}\left\|\frac{\partial \widetilde{Q}_{n}}{\partial \tilde{\sigma}_{0}}\right\|_{\infty} .
$$

Let us write for short $\widetilde{Y}=\widetilde{Y}_{n}$ and $\widetilde{Q}=\widetilde{Q}_{n}$, and set

$$
X=X\left(x_{0}, s\right)=x_{0}+s \vec{n}\left(x_{0}\right)+s^{2} \widetilde{Y}\left(x_{0}, s\right) .
$$

By (5.16),

$$
\begin{aligned}
\frac{\partial}{\partial \tilde{\sigma}_{0}}\left(T_{2}(\widetilde{Y}, \widetilde{Q})\right)\left(x_{0}, r\right)= & -T_{2}(\widetilde{Y}, \widetilde{Q}) \frac{a_{\tilde{\sigma}_{0}}\left(x_{0}\right)}{a\left(x_{0}\right)} \\
& +\frac{1}{2 a\left(x_{0}\right) r^{2}} \int_{0}^{r} \frac{1}{s} \frac{\partial}{\partial \tilde{\sigma}_{0}}\left(\nabla W\left(X\left(x_{0}, s\right)\right)-2 a\left(x_{0}\right) s \vec{n}\left(x_{0}\right)\right) d s \\
:= & A_{n}+B_{n} .
\end{aligned}
$$


Since the sequence $\left\{\left(\widetilde{Y}_{n}, \widetilde{Q}_{n}\right)\right\}_{n=0}^{\infty}$ converges uniformly, by Lemma 5.1, it follows that

$$
\left|A_{n}\right| \leq K_{1}, \forall n
$$

Next we compute

$$
\begin{aligned}
\frac{\partial}{\partial \tilde{\sigma}_{0}} & \nabla W\left(X\left(x_{0}, s\right)\right) \\
& =\frac{\partial \nabla W}{\partial \tilde{\sigma}}\left(X\left(x_{0}, s\right)\right) \frac{\partial \tilde{\sigma}\left(X\left(x_{0}, s\right)\right)}{\partial \tilde{\sigma}_{0}}+\frac{\partial \nabla W}{\partial \tilde{\delta}}\left(X\left(x_{0}, s\right)\right) \frac{\partial \tilde{\delta}\left(X\left(x_{0}, s\right)\right)}{\partial \tilde{\sigma}_{0}} \\
& =\left(\frac{\partial \nabla W}{\partial \tilde{\sigma}} \nabla \tilde{\sigma}+\frac{\partial \nabla W}{\partial \tilde{\delta}} \nabla \tilde{\delta}\right)\left(X\left(x_{0}, s\right)\right) \cdot\left(\left(1+s c\left(x_{0}\right)\right) \vec{\tau}\left(x_{0}\right)+s^{2} \frac{\partial \tilde{Y}}{\partial \tilde{\sigma}_{0}}\right) .
\end{aligned}
$$

Denoting

$$
Z=Z\left(x_{0}, s\right)=\left(\frac{1}{s}+c\left(x_{0}\right)\right) \vec{\tau}\left(x_{0}\right)+s \frac{\partial \widetilde{Y}}{\partial \tilde{\sigma}_{0}},
$$

we get from (5.25) and (5.3)-(5.4):

$$
\begin{aligned}
& \frac{1}{s} \frac{\partial}{\partial \tilde{\sigma}_{0}}(\nabla W(X))-\frac{\partial}{\partial \tilde{\sigma}_{0}}\left(2 a\left(x_{0}\right) \vec{n}\left(x_{0}\right)\right) \\
& =\left[\left(a_{\tilde{\sigma} \tilde{\sigma}} \tilde{\delta}^{2}+c_{1}(X)\left(a_{\tilde{\delta}} \tilde{\delta}^{2}+2 a \tilde{\delta}\right)+c_{3}(X) a_{\tilde{\sigma}} \tilde{\delta}^{2}\right) \vec{\tau}(X)\right. \\
& \left.+\left(\left(a_{\tilde{\sigma} \tilde{\delta}}+c_{2}(X) a_{\tilde{\sigma}}\right) \tilde{\delta}^{2}+2 a_{\tilde{\sigma}} \tilde{\delta}\right) \vec{n}(X)\right] \\
& \quad \cdot(Z \cdot \vec{\tau}(X))+\left[\left(a_{\tilde{\sigma} \tilde{\delta}} \tilde{\delta}^{2}+2 a_{\tilde{\sigma}} \tilde{\delta}+c_{4}(X) a_{\tilde{\sigma}} \tilde{\delta}^{2}\right) \vec{\tau}(X)\right. \\
& \left.+\left(a_{\tilde{\delta} \tilde{\delta}} \tilde{\delta}^{2}+4 a_{\tilde{\delta}} \tilde{\delta}+2 a\right) \vec{n}(X)\right](Z \cdot \vec{n}(X)) \\
& \quad-2 a_{\tilde{\sigma}_{0}}\left(x_{0}\right) \vec{n}\left(x_{0}\right)-2 a\left(x_{0}\right) c\left(x_{0}\right) \vec{\tau}\left(x_{0}\right) .
\end{aligned}
$$

Using the estimates,

$$
\vec{\tau}(X) \cdot \vec{\tau}\left(x_{0}\right)=1+O\left(s^{2}\right), \vec{n}(X) \cdot \vec{\tau}\left(x_{0}\right)=O\left(s^{2}\right), \tilde{\delta}(X)=s+O\left(s^{2}\right),
$$

together with the regularity of the functions $a(x)\left(C^{2}\right)$ and $c(x)\left(C^{1}\right)$ in (5.27), yields

$$
\left|\frac{1}{s} \frac{\partial}{\partial \tilde{\sigma}_{0}}(\nabla W(X))-\frac{\partial}{\partial \tilde{\sigma}_{0}}\left(2 a\left(x_{0}\right) \vec{n}\left(x_{0}\right)\right)\right| \leq K_{2} s+\left\|\frac{\partial \widetilde{Y}}{\partial \tilde{\sigma}_{0}}\right\|_{\infty}\left(2 a(X) s+K_{3} s^{2}\right) .
$$

It follows that for $R \leq R_{0}^{\prime}$ there holds

$$
\begin{aligned}
\left|B_{n}\right| & \leq \frac{1}{2 a\left(x_{0}\right) r^{2}} \int_{0}^{r} K_{2} s d s+\left\|\frac{\partial \widetilde{Y}}{\partial \tilde{\sigma}_{0}}\right\|_{\infty} \frac{1}{2 a\left(x_{0}\right) r^{2}} \int_{0}^{r}\left(2 a(X) s+K_{3} s^{2}\right) d s \\
& \leq K_{4}+\frac{3}{4}\left\|\frac{\partial \widetilde{Y}}{\partial \tilde{\sigma}_{0}}\right\|_{\infty},
\end{aligned}
$$

for some positive constant $K_{4}$. Combining the estimate (5.28) with (5.24), (5.21) and (5.23) we obtain that

$$
\max \left(\left\|\frac{\partial \widetilde{Y}_{n+1}}{\partial \tilde{\sigma}_{0}}\right\|_{\infty},\left\|\frac{\partial \widetilde{Q}_{n+1}}{\partial \tilde{\sigma}_{0}}\right\|_{\infty}\right) \leq \max \left(K_{1}, K_{4}\right)+\frac{3}{4} \max \left(\left\|\frac{\partial \widetilde{Y}_{n}}{\partial \tilde{\sigma}_{0}}\right\|_{\infty},\left\|\frac{\partial \widetilde{Q}_{n}}{\partial \tilde{\sigma}_{0}}\right\|_{\infty}\right),
$$

which clearly implies (5.20). 
Next, let $(\widetilde{Y}, \widetilde{Q})$ and $\left(\widetilde{Y}^{\prime}, \widetilde{Q}^{\prime}\right)$ be $C^{1}$ functions defined on $\Gamma \times[0, R]$ for $R$ small enough, to be determined later, and let $X$ and $X^{\prime}$ be associated to $\widetilde{Y}$ and $\widetilde{Y}^{\prime}$, respectively. Let the corresponding $Z$ and $Z^{\prime}$ be defined by (5.26). Note first that the equality

implies that

$$
\frac{\partial}{\partial \tilde{\sigma}_{0}}\left(T_{1}(\widetilde{Y}, \widetilde{Q})\right)=\frac{1}{r^{2}} \int_{0}^{r} s \frac{\partial \widetilde{Q}}{\partial \tilde{\sigma}_{0}}\left(x_{0}, s\right) d s,
$$

$$
\left\|\frac{\partial}{\partial \tilde{\sigma}_{0}} T_{1}(\widetilde{Y}, \widetilde{Q})-\frac{\partial}{\partial \tilde{\sigma}_{0}} T_{1}\left(\widetilde{Y}^{\prime}, \widetilde{Q}^{\prime}\right)\right\|_{\infty} \leq \frac{1}{2}\left\|\frac{\partial \widetilde{Q}}{\partial \tilde{\sigma}_{0}}-\frac{\partial \widetilde{Q}^{\prime}}{\partial \tilde{\sigma}_{0}}\right\|_{\infty}
$$

From (5.23) we get that

$$
\begin{aligned}
& \left\|\frac{\partial}{\partial \tilde{\sigma}_{0}} T_{2}(\widetilde{Y}, \widetilde{Q})-\frac{\partial}{\partial \tilde{\sigma}_{0}} T_{2}\left(\widetilde{Y}^{\prime}, \widetilde{Q}^{\prime}\right)\right\|_{\infty} \leq c_{0}\left\|\widetilde{Q}-\widetilde{Q}^{\prime}\right\|_{\infty} \\
& \quad+\frac{1}{2 a\left(x_{0}\right) r^{2}} \int_{0}^{r} \frac{1}{s}\left\|\frac{\partial}{\partial \tilde{\sigma}_{0}} \nabla W\left(X\left(x_{0}, s\right)\right)-\frac{\partial}{\partial \tilde{\sigma}_{0}} \nabla W\left(X^{\prime}\left(x_{0}, s\right)\right)\right\|_{\infty} d s .
\end{aligned}
$$

Next we estimate for each $s \in(0, r)$,

$$
\begin{aligned}
\frac{1}{s} \| & \frac{\partial}{\partial \tilde{\sigma}_{0}} \nabla W(X)-\frac{\partial}{\partial \tilde{\sigma}_{0}} \nabla W\left(X^{\prime}\right) \|_{\infty} \\
=\|\left(\frac{\partial \nabla W}{\partial \tilde{\sigma}} \nabla \tilde{\sigma}\right. & \left.+\frac{\partial \nabla W}{\partial \tilde{\delta}} \nabla \tilde{\delta}\right)(X) \cdot Z-\left(\frac{\partial \nabla W}{\partial \tilde{\sigma}} \nabla \tilde{\sigma}+\frac{\partial \nabla W}{\partial \tilde{\delta}} \nabla \tilde{\delta}\right)\left(X^{\prime}\right) \cdot Z^{\prime} \|_{\infty} \\
\leq \|\left(\frac{\partial \nabla W}{\partial \tilde{\sigma}} \nabla \tilde{\sigma}\right. & \left.+\frac{\partial \nabla W}{\partial \tilde{\delta}} \nabla \tilde{\delta}\right)(X)-\left(\frac{\partial \nabla W}{\partial \tilde{\sigma}} \nabla \tilde{\sigma}+\frac{\partial \nabla W}{\partial \tilde{\delta}} \nabla \tilde{\delta}\right)\left(X^{\prime}\right)\left\|_{\infty}\right\| Z \|_{\infty} \\
& +\left\|\left(\frac{\partial \nabla W}{\partial \tilde{\sigma}} \nabla \tilde{\sigma}+\frac{\partial \nabla W}{\partial \tilde{\delta}} \nabla \tilde{\delta}\right)\left(X^{\prime}\right)\right\|_{\infty}\left\|Z-Z^{\prime}\right\|_{\infty}:=C_{n}+D_{n} .
\end{aligned}
$$

Since $W$ is of class $C^{3}$ we get, for some constants $c, c_{1}, c_{2}, C$, that

$$
\begin{array}{r}
\left|C_{n}\right| \leq c\left\|X-X^{\prime}\right\|_{\infty}\|Z\|_{\infty} \leq c_{1} s^{2}\left\|\frac{\partial \tilde{Y}}{\partial \tilde{\sigma}_{0}}-\frac{\partial \tilde{Y}^{\prime}}{\partial \tilde{\sigma}_{0}}\right\|_{\infty}\left(\frac{1}{s}+c_{2}+s\left\|\frac{\partial \tilde{Y}}{\partial \tilde{\sigma}_{0}}\right\|_{\infty}\right) \\
\leq C s\left\|\frac{\partial \tilde{Y}}{\partial \tilde{\sigma}_{0}}-\frac{\partial \tilde{Y}^{\prime}}{\partial \tilde{\sigma}_{0}}\right\|_{\infty},
\end{array}
$$

with $C$ depending on $\left\|\frac{\partial \tilde{Y}}{\partial \tilde{\sigma}_{0}}\right\|_{\infty}$. Furthermore, by (5.3)-(5.4) we have

$$
\left|D_{n}\right| \leq\left(2 a\left(X^{\prime}\right)+c_{3} s\right) s\left\|\frac{\partial \tilde{Y}}{\partial \tilde{\sigma}_{0}}-\frac{\partial \tilde{Y}^{\prime}}{\partial \tilde{\sigma}_{0}}\right\|_{\infty} \leq\left(2 a\left(x_{0}\right)+c_{4} s\right) s\left\|\frac{\partial \tilde{Y}}{\partial \tilde{\sigma}_{0}}-\frac{\partial \tilde{Y}^{\prime}}{\partial \tilde{\sigma}_{0}}\right\|_{\infty},
$$

with $c_{3}, c_{4}$ depending on the $L^{\infty}$-norm of $\tilde{Y}^{\prime}$. Using (5.31)-(5.32) in (5.30), we obtain that

$\left\|\frac{\partial}{\partial \tilde{\sigma}_{0}}\left(T_{2}(\widetilde{Y}, \widetilde{Q})-T_{2}\left(\widetilde{Y}^{\prime}, \widetilde{Q}^{\prime}\right)\right)\right\|_{\infty} \leq c_{0}\left\|\widetilde{Q}-\widetilde{Q}^{\prime}\right\|_{\infty}+(1 / 2+O(R))\left\|\frac{\partial \widetilde{Y}}{\partial \tilde{\sigma}_{0}}-\frac{\partial \widetilde{Y}^{\prime}}{\partial \tilde{\sigma}_{0}}\right\|_{\infty}$.

Next we apply the above estimates for the choice $(\widetilde{Y}, \widetilde{Q})=\left(\widetilde{Y}_{n}, \widetilde{Q}_{n}\right)$ and $\left(\widetilde{Y}^{\prime}, \widetilde{Q}^{\prime}\right)$ $=\left(\widetilde{Y}_{n+1}, \widetilde{Q}_{n+1}\right)$. Using Lemma 5.1 and (5.20) we conclude from (5.33) that for $R$ small enough we have

$$
\left\|\frac{\partial}{\partial \tilde{\sigma}_{0}}\left(\tilde{Y}_{n+2}-\tilde{Y}_{n+1}\right)\right\|_{\infty} \leq c_{0}\left\|\widetilde{Q}_{n}-\widetilde{Q}_{n+1}\right\|_{\infty}+\frac{7}{8}\left\|\frac{\partial}{\partial \tilde{\sigma}_{0}}\left(\widetilde{Y}_{n}-\tilde{Y}_{n+1}\right)\right\|_{\infty} .
$$


On the other hand, the estimate (5.29) now reads

$$
\left\|\frac{\partial}{\partial \tilde{\sigma}_{0}}\left(\widetilde{Q}_{n+2}-\widetilde{Q}_{n+1}\right)\right\|_{\infty} \leq \frac{1}{2}\left\|\frac{\partial}{\partial \tilde{\sigma}_{0}}\left(\widetilde{Q}_{n+1}-\widetilde{Q}_{n}\right)\right\|_{\infty} .
$$

Combining (5.34) with (5.35) and using (5.19) we easily conclude the convergence of the sequence $\left\{\left(\frac{\partial}{\partial \tilde{\sigma}_{0}} \widetilde{Y}_{n}, \frac{\partial}{\partial \tilde{\sigma}_{0}} \widetilde{Q}_{n}\right)\right\}$ in the $L^{\infty}$-norm, and the result follows.

Using the above lemmata we can now present the proof of Proposition 5.1.

Proof of Proposition [5.1. Denote by $(\widetilde{Y}, \widetilde{Q})=\left(\widetilde{Y}_{\infty}, \widetilde{Q}_{\infty}\right)$ the solution constructed in Lemma 5.1, Put

$$
\begin{aligned}
& X\left(x_{0}, s\right)=x_{0}+s \vec{n}\left(x_{0}\right)+s^{2} \widetilde{Y}\left(x_{0}, s\right), \\
& P\left(x_{0}, s\right)=\sqrt{a\left(x_{0}\right)}\left(s \vec{n}\left(x_{0}\right)+s^{2} \widetilde{Q}\left(x_{0}, s\right)\right), \quad \forall x_{0} \in \Gamma, \forall s \in[-R, R] .
\end{aligned}
$$

Note that

and

$$
\frac{\partial X}{\partial \tilde{\sigma}_{0}}\left(x_{0}, s\right)=\vec{\tau}\left(x_{0}\right)+s c\left(x_{0}\right) \vec{\tau}\left(x_{0}\right)+s^{2} \frac{\partial \widetilde{Y}}{\partial \tilde{\sigma}_{0}}\left(x_{0}, s\right)
$$

$$
\frac{\partial X}{\partial s}\left(x_{0}, s\right)=\frac{2 P\left(x_{0}, s\right)}{2 s \sqrt{a\left(x_{0}\right)}}=\vec{n}\left(x_{0}\right)+s \widetilde{Q}\left(x_{0}, s\right)(\text { see }(\underline{5.8}))
$$

are continuous by Lemmata 5.1 and 5.2. In particular,

$$
\frac{\partial X}{\partial \tilde{\sigma}_{0}}\left(x_{0}, 0\right)=\vec{\tau}\left(x_{0}\right) \text { and } \frac{\partial X}{\partial s}\left(x_{0}, 0\right)=\vec{n}\left(x_{0}\right),
$$

and it follows that $D X\left(x_{0}, 0\right)$ is nonsingular. Therefore, by the implicit function theorem there exists a neighborhood $\Omega_{0}$ of $\Gamma$, and $0<R_{2} \leq R_{1}$ (see Lemma 5.2) such that $X: \Gamma \times\left(-R_{2}, R_{2}\right) \rightarrow \Omega_{0}$ is a $C^{1}$-diffeomorphism. We finally define $U$ in $\Omega_{0}$ by

$$
U(X)=U\left(X\left(x_{0}, r\right)\right)=\int_{0}^{r} P\left(X\left(x_{0}, s\right)\right) \cdot \frac{\partial X}{\partial s}\left(x_{0}, s\right) d s .
$$

By (5.6) and (5.8) we have

$$
\frac{\partial}{\partial r} H(X, P)=\frac{\partial H}{\partial X} \cdot \frac{\partial X}{\partial r}+\frac{\partial H}{\partial P} \cdot \frac{\partial P}{\partial r}=\frac{1}{\alpha r}(-\nabla W \cdot 2 P+2 P \cdot \nabla W)=0,
$$

which implies that

$$
H\left(X\left(x_{0}, r\right), P\left(x_{0}, r\right)\right)=H\left(x_{0}, 0\right)=0, \forall x_{0}, \forall r .
$$

If we can show that

$$
\nabla U=P,
$$

it will follow from (5.37) that $U$ solves (5.5). For that matter we differentiate (5.36) and get:

and

$$
\frac{\partial U}{\partial r}=\nabla U \cdot \frac{\partial X}{\partial r}=P \cdot \frac{\partial X}{\partial r}
$$

Differentiating (5.37) w.r.t. $\tilde{\sigma}_{0}$ yields

$$
\frac{\partial U}{\partial \tilde{\sigma}_{0}}=\nabla U \cdot \frac{\partial X}{\partial \tilde{\sigma}_{0}}=\int_{0}^{r}\left[\frac{\partial P}{\partial \tilde{\sigma}_{0}} \cdot \frac{\partial X}{\partial s}+P \cdot \frac{\partial^{2} X}{\partial s \partial \tilde{\sigma}_{0}}\right] d s .
$$

$$
\frac{\partial H}{\partial X} \cdot \frac{\partial X}{\partial \tilde{\sigma}_{0}}+\frac{\partial H}{\partial P} \cdot \frac{\partial P}{\partial \tilde{\sigma}_{0}}=0
$$


Using (5.41) we get that

(5.42)

$\frac{\partial P}{\partial \tilde{\sigma}_{0}} \cdot \frac{\partial X}{\partial s}=\frac{\partial P}{\partial \tilde{\sigma}_{0}} \cdot \frac{2 P}{\alpha s}=\frac{1}{\alpha s} \frac{\partial H}{\partial P} \cdot \frac{\partial P}{\partial \tilde{\sigma}_{0}}=-\frac{1}{\alpha s} \frac{\partial H}{\partial X} \cdot \frac{\partial X}{\partial \tilde{\sigma}_{0}}=\frac{\nabla W}{\alpha s} \cdot \frac{\partial X}{\partial \tilde{\sigma}_{0}}=\frac{\partial P}{\partial s} \cdot \frac{\partial X}{\partial \tilde{\sigma}_{0}}$.

Plugging (5.42) in (5.40) yields

$\left.\nabla U \cdot \frac{\partial X}{\partial \tilde{\sigma}_{0}}=\int_{0}^{r}\left[\frac{\partial P}{\partial s} \cdot \frac{\partial X}{\partial \tilde{\sigma}_{0}}+P \cdot \frac{\partial^{2} X}{\partial s \partial \tilde{\sigma}_{0}}\right] d s=P \cdot \frac{\partial X}{\partial \tilde{\sigma}_{0}}\right]_{0}^{r}=P\left(x_{0}, r\right) \cdot \frac{\partial X}{\partial \tilde{\sigma}_{0}}\left(x_{0}, r\right)$.

In a neighborhood of $\Gamma$ the vectors $\frac{\partial X}{\partial \tilde{\sigma}_{0}}$ and $\frac{\partial X}{\partial s}$ are linearly independent, so (5.38) follows from (5.39) and (5.43). We conclude that $U$ is a solution of (5.5) in some neighborhood $\Gamma_{\eta_{1}}$ of $\Gamma$ and the $C^{2}$-regularity of $U$ follows from the regularity of $X$ and $P$.

Finally we prove that $U$ coincides with $\Psi$ in $\Gamma_{\eta_{1}}$. Fix a point $\zeta \in \Gamma_{\eta_{1}}$ and take any Lipschitz map $\gamma:[0,1] \rightarrow \mathbb{R}^{2}$ such that $\gamma(0) \in \Gamma$ and $\gamma(1)=\zeta$. Then,

$$
\begin{aligned}
\int_{0}^{1}(W(\gamma(t)))^{1 / 2}\left|\gamma^{\prime}(t)\right| d t & =\int_{0}^{1}|\nabla U(\gamma(t))|\left|\gamma^{\prime}(t)\right| d t \\
& \geq \int_{0}^{1} \nabla U(\gamma(t)) \cdot \gamma^{\prime}(t) d t=U(\gamma(1))-U(\gamma(0))=U(\zeta) .
\end{aligned}
$$

Therefore, $\Psi(\zeta) \geq U(\zeta)$. On the other hand, there exists a unique $x_{0} \in \Gamma$ such that $\zeta=X\left(x_{0}, r(\zeta)\right)$ for some $r(\zeta)>0$. Then we can define a path connecting $x_{0}$ to $\zeta$ by

$$
\gamma_{0}(s)=X\left(x_{0}, s\right), s \in[0, r(\zeta)] .
$$

Since $\nabla U\left(\gamma_{0}(s)\right)=P\left(x_{0}, s\right)$ by (5.38), $\sqrt{W\left(\gamma_{0}(s)\right)}=\left|P\left(x_{0}, s\right)\right|$ by (5.37) and $\gamma_{0}^{\prime}(s)=\frac{2 P\left(x_{0}, s\right)}{\alpha s}$ by (5.8), we have

$$
\begin{gathered}
\int_{0}^{r(\zeta)}\left(W\left(\gamma_{0}(s)\right)\right)^{1 / 2}\left|\gamma_{0}^{\prime}(s)\right| d s=\int_{0}^{r(\zeta)}\left|P\left(x_{0}, s\right)\right| \frac{\left|2 P\left(x_{0}, s\right)\right|}{\alpha s} d s \\
=\int_{0}^{r(\zeta)} \nabla U\left(\gamma_{0}(s)\right) \cdot \gamma_{0}^{\prime}(s) d s=U\left(\gamma_{0}(r(\zeta))\right)-U\left(\gamma_{0}(0)\right)=U(\zeta),
\end{gathered}
$$

which implies that $U(\zeta) \geq \Psi(\zeta)$. Combining the two inequalities yields $U(\zeta)=\Psi(\zeta)$ as claimed.

\section{ACKNOWLEDGMENT}

The authors are grateful to Professor H. Brezis for suggesting to them the problem studied in this article and for his constant interest. They also thank Prof. G. Barles for interesting discussions and suggestions concerning the problem treated in the Appendix. The research of I. Shafrir was supported by the fund for the promotion of research at the Technion.

\section{REFERENCES}

[1] N. André and I. Shafrir, Minimization of a Ginzburg-Landau type functional with nonvanishing Dirichlet boundary condition, Calc. Var. Partial Differential Equations 7 (1998), 191-217. MR.1651415 (99k:35161)

[2] N. André and I. Shafrir, On a singular perturbation problem involving the distance to a curve, J. d'Anal. Math. 90 (2003), 337-396. MR2001075 (2004h:35052) 
[3] N. André and I. Shafrir, On the minimizers of a Ginzburg-Landau type energy when the boundary condition has zeros, Advances in Diff. Equations 9 (2004), 891-960. MR2100398 (2005g:35050)

[4] F. Bethuel, H. Brezis and F. Hélein, Asymptotics for the minimization of a GinzburgLandau functional, Calc. Var. Partial Differential Equations 1 (1993),123-148. MR:1261720 (94m:35083)

[5] F. Bethuel, H. Brezis and F. Hélein, Ginzburg-Landau Vortices, Birkhäuser, 1994. MR.1269538 (95c:58044)

[6] L. C. Evans, Partial Differential Equations, Graduate Studies in Mathematics, 19. American Mathematical Society, Providence, RI, 1998. MR.1625845 (99e:35001)

[7] I. Fonseca and L. Tartar, The gradient theory of phase transitions for systems with two potential wells, Proc. Roy. Soc. Edinburgh Sect. A 111 (1989), 89-102. MR985992 (90b:49021)

[8] D. Gilbarg and N. Trudinger, Elliptic Partial Differential Equations of Second Order, Springer-Verlag, Berlin and New York, 1983. MR.737190 (86c:35035)

[9] L. Lassoued and P. Mironescu, Ginzburg-Landau type energy with discontinuous constraint, J. Anal. Math. 77 (1999), 1-26. MR1753480(2001e:58014)

[10] L. Modica, The gradient theory of phase transitions and the minimal interface criterion, Arch. Rational Mech. Anal. 98 (1987), 123-142. MR866718 (88f:76038)

[11] P. Sternberg, The effect of a singular perturbation on nonconvex variational problems, Arch. Rational Mech. Anal. 101 (1988), 209-260. MR930124 (89h:49007)

[12] M. Struwe, On the asymptotic behavior of minimizers of the Ginzburg-Landau model in 2 dimensions, Differential Integral Equations 7 (1994), 1613-1624; erratum, loc. cit. 8 (1995), 124. MR1269674 (95g:35057a)

Département de Mathématiques, Université de Tours, 37200 Tours, France

Department of Mathematics, Technion - Israel Institute of Technology, 32000 Haifa, ISRAEL 